\title{
TREM2 expression in the brain and biological fluids in prion diseases
}

\author{
Daniela Diaz-Lucena ${ }^{1,2} \cdot$ Niels Kruse $^{3} \cdot$ Katrin Thüne $^{4} \cdot$ Matthias Schmitz $^{4,5} \cdot$ Anna Villar-Piqué $^{1,2}$. \\ Jose Eriton Gomes da Cunha ${ }^{6}$. Peter Hermann ${ }^{4}$. Óscar López-Pérez ${ }^{1,2}$. Pol Andrés-Benito ${ }^{1,2}$. Anna Ladogana ${ }^{7}$. \\ Miguel Calero ${ }^{8,9}$. Enric Vidal ${ }^{10}$. Joachim Riggert ${ }^{11}$. Hailey Pineau ${ }^{12}$. Valerie Sim ${ }^{12}$. Henrik Zetterberg ${ }^{13,14,15,16}$. \\ Kaj Blennow ${ }^{13,14}$. Jose Antonio del Río ${ }^{17,18,19,20}$. Alba Marín-Moreno ${ }^{21}$. Juan Carlos Espinosa ${ }^{21}$. \\ Juan María Torres ${ }^{21} \cdot$ Raquel Sánchez-Valle ${ }^{22} \cdot$ Brit Mollenhauer $^{23,24} \cdot$ Isidre Ferrer $^{1,2,25}$ (D) Inga Zerr ${ }^{4,5}$. \\ Franc Llorens ${ }^{1,2,4}$
}

Received: 23 February 2021 / Revised: 15 March 2021 / Accepted: 15 March 2021 / Published online: 21 April 2021 (c) The Author(s) 2021

\begin{abstract}
Triggering receptor expressed on myeloid cells 2 (TREM2) is an innate immune cell surface receptor that regulates microglial function and is involved in the pathophysiology of several neurodegenerative diseases. Its soluble form (sTREM2) results from shedding of the TREM 2 ectodomain. The role of TREM 2 in prion diseases, a group of rapidly progressive dementias remains to be elucidated. In the present study, we analysed the expression of TREM 2 and its main sheddase ADAM10 in the brain of sporadic Creutzfeldt-Jakob disease (sCJD) patients and evaluated the role of CSF and plasma sTREM2 as a potential diagnostic marker of prion disease. Our data indicate that, compared to controls, TREM2 is increased in sCJD patient brains at the mRNA and protein levels in a regional and subtype dependent fashion, and expressed in a subpopulation of microglia. In contrast, ADAM10 is increased at the protein, but not the mRNA level, with a restricted neuronal expression. Elevated CSF sTREM2 is found in SCJD, genetic CJD with mutations E200K and V210I in the prion protein gene (PRNP), and iatrogenic $\mathrm{CJD}$, as compared to healthy controls $(\mathrm{HC})(\mathrm{AUC}=0.78-0.90)$ and neurological controls $(\mathrm{AUC}=0.73-0.85)$, while CSF sTREM2 is unchanged in fatal familial insomnia. sTREM2 in the CSF of cases with Alzheimer's disease, and multiple sclerosis was not significantly altered in our series. CSF sTREM2 concentrations in SCJD are PRNP codon 129 and subtype-related, correlate with CSF 14-3-3 positivity, total-tau and YKL-40, and increase with disease progression. In plasma, sTREM2 is increased in SCJD compared with $\mathrm{HC}(\mathrm{AUC}=0.80)$, displaying positive correlations with plasma total-tau, neurofilament light, and YKL-40. We conclude that comparative study of TREM2 in brain and biological fluids of prion diseases reveals TREM2 to be altered in human prion diseases with a potential value in target engagement, patient stratification, and disease monitoring.
\end{abstract}

Keywords TREM $2 \cdot$ Prion diseases $\cdot$ Creutzfeldt-Jakob disease $\cdot$ Microglia $\cdot$ Cerebrospinal fluid $\cdot$ Plasma

\section{Introduction}

Prion diseases (or transmissible spongiform encephalopathies) are a rare group of invariably fatal neurodegenerative dementias characterized by a rapid progression affecting humans and animals. The causative agent of prion diseases is the pathological form of the cellular prion protein

Isidre Ferrer

80982ifa@gmail.com

Inga Zerr

ingazerr@med.uni-goettingen.de

Extended author information available on the last page of the article
$\left(\operatorname{PrP}^{\mathrm{c}}\right), \operatorname{Pr} \mathrm{P}^{\mathrm{Sc}}$, which nucleates and self-propagates in the central nervous system (CNS) [3]. However, the mechanisms by which $\mathrm{PrP}^{\mathrm{Sc}}$ causes neurodegeneration are not well-understood.

Human prion diseases can be classified according to their aetiology as sporadic Creutzfeldt-Jakob disease (sCJD); genetic Creutzfeldt-Jakob disease (gCJD), Gerstmann-Sträussler-Scheinker syndrome (GSS), and fatal familial insomnia (FFI); and as iatrogenic Creutzfeldt-Jakob disease (iCJD), variant Creutzfeldt-Jakob disease (vCJD), and Kuru. sCJD is the most prevalent human prion disease and its typical clinical phenotype includes rapidly progressive dementia, behavioural abnormalities, ataxia, extrapyramidal features, 
and myoclonus. Eventually, sCJD patients fall into a state of akinetic mutism before death $[18,54]$. Neuropathologically, SCJD is characterized by neuronal loss, spongiform change, reactive astrocytosis, and microgliosis. These changes are accompanied by the accumulation of a protease-resistant form of host-derived prion protein $\left(\mathrm{PrP}^{\mathrm{res}}\right)$ [17].

A clinical diagnosis of probable SCJD is achieved by the combination of clinical symptoms and signs, neuroimaging, and cerebrospinal fluid tests (CSF) $[23,26]$. The most relevant CSF biomarkers are surrogate neuronal damage markers 14-3-3, total-tau (t-tau), and $\alpha$-synuclein (a-syn), while the real-time quaking induced conversion (RT-QuIC) assay is a prion-specific assay allowing identification of the pathological prion protein in the CSF [70]. Recently, some blood biomarkers have been shown to be suitable in discriminating sCJD cases but their accuracy in the differential diagnosis of sCJD has not been determined [30, 66, 72]. Therefore, they are not yet included in clinical practice. Importantly, the use of surrogate CSF biomarkers is not limited to diagnostic purposes; rather, a potential use as prognostic, predictive, monitoring, and response biomarkers has been proposed [35, 46, $65,71]$. Therefore, the characterization of the best putative application for each biomarker as well as the identification of biomarkers targeting the complete pathological signatures of prion disease will greatly enhance the rational development and interpretation of future therapeutic interventions. In this regard, the observation that exacerbated inflammatory profiles is an early pathogenic event in prion diseases $[2,34]$ has led to the search for surrogate biomarkers of neuroinflammation for early diagnosis. In this context, glialspecific proteins such as the $\mathrm{S} 100$ calcium-binding protein B (S100B), chitinase-3-like protein 1 (CHI3L1/YKL-40), chitotriosidase 1 (CHIT1), and the glial fibrillary acidic protein (GFAP) have been reported to be increased in the CSF of sCJD [2, 25, 37, 58].

In recent years, strong evidence of a link between TREM2 function in the innate immune system and the pathogenesis of neurodegenerative diseases has been documented. Although rare in the population, certain TREM2 variants are associated with increased risk of developing neurodegenerative diseases such as Alzheimer's disease (AD) [21, 26], frontotemporal dementia [20, 56], Parkinson's disease [56], and amyotrophic lateral sclerosis [7], presumably as a consequence of TREM2 loss of function [12]. In this regard, TREM2 mutations led to reduced phagocytic function [64], while injection of sTREM2 in an AD mouse model enhanced microglial proliferation, migration, clustering in the vicinity of amyloid plaques, and the uptake and degradation of $\beta$-amyloid [79]. In AD patients, increased TREM2 levels have been reported in the CSF [22, 24, 41, 42, 55], together with positive immunoreactivity in the microglia associated with $\beta$-amyloid plaques and in neuritic pathologyenriched areas [41]. It is currently accepted that TREM2 is expressed predominantly in cells of myeloid lineage, in line with its role in brain innate immune function [15, 28, 61]. No effect of genetic variation in TREM2 in the risk of CJD has been identified [63]. Yet pioneering studies have shown increased microglial TREM2 mRNA expression in scrapieinfected mice and prion-inoculated TREM2 knockout mice, with attenuated microglia response compared to controls but no alterations in prion pathogenesis in terms of disease onset, disease duration, and presence of pathological PrP [80]. TREM 2 expression in brain and biological fluids of human prion diseases and its potential role as a candidate biomarker of prion pathogenesis have not been explored.

\section{Material and methods}

\section{Antibodies and reagents}

Human TREM2 goat polyclonal antibody (RD Systems, AF1828) was used diluted 1:20 for immunohistochemistry and at a dilution of 1:200 for western blotting. The antibody anti-TREM2 Abcam [EPR20243] ab209814 diluted 1:100 was used for immunohistochemistry for comparative purposes. Human ADAM10 antibody (Abcam, ab1997) was used at a dilution of 1:75 for immunohistochemistry and 1:500 for western blotting. Monoclonal anti- $\beta$-actin antibody (Sigma-Aldrich, A5316) was used at 1:30,000 as a proteinloading control in western blotting. For double-labelling immunofluorescence and confocal microscopy visualization, TREM2 was combined with the microglial marker IBA1 diluted 1:1000 (Wako, 019-19,741).

\section{Brain samples}

Brain tissue was obtained from the Institute of Neuropathology HUB-ICO-IDIBELL Biobank and the Hospital Clinic de Barcelona-IDIBAPS Neurological Tissue Bank following the Spanish legislation guidelines on this matter (Real Decreto de Biobancos 1716/2011) and approval of the local ethics committees. Processing of brain tissue has been detailed elsewhere [16]. The post-mortem interval between death and tissue processing was between $1 \mathrm{~h} 30 \mathrm{~min}$ and $24 \mathrm{~h}$. One hemisphere was immediately cut in coronal sections, $1-\mathrm{cm}$ thick, and selected areas of the encephalon were rapidly dissected, frozen on metal plates over dry ice, placed in individual air-tight plastic bags, numbered with water-resistant ink, and stored at $-80{ }^{\circ} \mathrm{C}$ until use for biochemical studies. The other hemisphere was fixed by immersion in $4 \%$ buffered formalin for 3 weeks for morphologic study. Neuropathological examination was performed in every case on formalin-fixed, paraffin-embedded samples. De-waxed paraffin Sects. 4 microns thick were stained with haematoxylin and eosin, periodic acid Schiff, Congo red, 
and Klüver-Barrera, or processed for immunohistochemistry using anti- $\beta$-amyloid, p-tau (clone AT8), $\alpha$-synuclein, $\alpha B$-crystallin, TDP-43, P-TDP-43, ubiquitin, p62, glial fibrillary acidic protein, CD68, and IBA1, as previously reported [16]. Neuropathological diagnosis of Alzheimer's disease (AD) was categorized following Braak stages of neurofibrillary tangle pathology adapted to paraffin sections [5, 6]. Cases with Frontotemporal lobar degeneration with TDP43 pathology (FTLD-TDP) were diagnosed following established neuropathological criteria [43]. Post-mortem neuropathological examination confirmed the diagnosis of SCJD cases according to established criteria $[49,50]$. The analysis of the codon129 genotype of PrP gene (PRNP) (methionine or valine) was performed after isolation of genomic DNA from blood samples according to standard methods. SCJD type 1 or type 2 classification was performed with western blotting. Cases with associated pathologies such as vascular diseases, infections of the nervous system, neoplastic diseases affecting the nervous system, systemic and central immune diseases, metabolic syndrome, hypoxia, and prolonged axonal states such as those occurring in intensive care units were excluded from the present study.

Since a percentage of CJD cases have concomitant NFT pathology at stages 0-III of Braak, with occasional amyloid plaques (stage A of Braak), control cases in the present series are not controls without NFT pathology but rather age-matched controls with NFT pathology at stages 0-III without neurological symptoms. Mild small blood vessel disease and occasional status cribosus occurred in the 'control' and 'pathological' groups.

Molecular studies were carried out in frontal cortex area $8(\mathrm{FC})$ and the cerebellum (CB). The CJD group was composed of controls ( $n=15 \mathrm{FC}, n=12 \mathrm{CB}), \operatorname{sCJD}(n=11$ $\mathrm{FC}, n=12 \mathrm{CB})$, and $\mathrm{SCJD} \operatorname{VV} 2(n=11 \mathrm{FC}, n=10 \mathrm{CB})$. The sFTLD group consisted of samples from the FC of controls $(n=17)$ and sFTLD-TDP $(n=16)$. Details of the brain samples used for q-PCR are summarized in Supplementary Table 1. Samples for western blotting were obtained from 10 controls, 8 sCJD MM1, and 8 sCJD VV2 cases.

Triggering receptor expressed on myeloid cells $2 \mathrm{immu}-$ nohistochemistry was performed on de-waxed paraffin sections of controls $(n=10 ; 7 \mathrm{M}, 3 \mathrm{~F}$; mean age \pm SD: $57.9 \pm 12.5)$, CJD MM1 $(n=7 ; 6 \mathrm{M}, 1$ not available; mean age \pm SD: $63.8 \pm 13.9)$, AD V-VI $(n=3 ; 3 \mathrm{~F}$; mean age \pm SD: $77.3 \pm 4.1)$, sFTLD-TDP $(n=3 ; 3 \mathrm{M}$; mean age \pm SD: $72 \pm 7.1)$, DLB ( $n=3 ; 2 \mathrm{~F}, 1 \mathrm{M}$; mean age \pm SD: $78.3 \pm 4.9)$, $\operatorname{ALS}(n=4 ; 3 \mathrm{~F}, 1 \mathrm{M}$; mean age $\pm \mathrm{SD}: 62 \pm 12.5)$, white matter with plaques of relapsing remitting cases of multiple sclerosis (MS, $n=1 \mathrm{M}, 52$ years), and vascular dementia with subacute areas of infarction (DM, $n=3 \mathrm{M}$; 72) (see Supplementary table 3).

\section{Cerebrospinal fluid and plasma samples}

Cerebrospinal fluid (CSF) samples (Table 1) from healthy control individuals (HC, $n=48$ ), patients with non-primarily neurodegenerative neurological diseases (ND, $n=64)$, AD $(n=35)$, and SCJD $(n=139)$ were collected at the Clinical Dementia Centre and the National Reference Centre for CJD Surveillance at the University Medical Centre of Göttingen (Germany) and at the Alzheimer's Disease and Other Cognitive Disorders Unit, Hospital Clínic de Barcelona, Barcelona (Spain). Patients with sCJD, classified as probable $(n=47)$ or definite $(n=92)$ cases according to diagnostic consensus

Table 1 Biomarker characteristics of the cases used in the CSF cohort of study. Diagnostic groups included controls (HC, ND), prion diseases (sCJD, gCJD-E200K, gCJD-V210I, FFI and iCJD), and AD cases

\begin{tabular}{|c|c|c|c|c|c|c|c|c|c|}
\hline & \multirow[t]{2}{*}{$n$} & \multirow[t]{2}{*}{$\operatorname{Sex}(f / m)$} & \multirow{2}{*}{$\begin{array}{l}\text { Age } \\
\text { Mean +SD }\end{array}$} & \multirow{2}{*}{$\begin{array}{l}\text { sTREM2 (pg/ } \\
\text { mL) } \\
\text { Mean + SD }\end{array}$} & \multirow[t]{2}{*}{$95 \% \mathrm{CI}$} & \multirow{2}{*}{$\begin{array}{l}\text { tau }(\mathrm{pg} / \mathrm{mL}) \\
\text { Mean + SD }\end{array}$} & \multirow{2}{*}{$\begin{array}{l}\text { 14-3-3 } \\
\text { Pos/neg }\end{array}$} & \multicolumn{2}{|l|}{$p$ value } \\
\hline & & & & & & & & $\begin{array}{l}\text { sTREM2 vs. } \\
\text { sex }\end{array}$ & sTREM2 vs. age \\
\hline $\mathrm{HC}$ & 48 & $26 / 22$ & $64 \pm 15$ & $1898 \pm 842$ & $1653-2143$ & $246 \pm 132$ & $0 / 48$ & 0.26 & 0.20 \\
\hline ND & 64 & $39 / 25$ & $66 \pm 9$ & $2321 \pm 1125$ & $2040-2602$ & $441 \pm 410$ & $13 / 49$ & 0.87 & 0.61 \\
\hline sCJD & 139 & $73 / 66$ & $66 \pm 11$ & $6975 \pm 8523$ & $5544-8403$ & $7337 \pm 7658^{*}$ & $118 / 21$ & 0.83 & 0.26 \\
\hline gCJD—E200K & 57 & $35 / 22$ & $60 \pm 10$ & $6341 \pm 8128$ & $4184-8498$ & $6548 \pm 5688$ & $49 / 8$ & 0.13 & 0.91 \\
\hline $\mathrm{gCJD}-\mathrm{V} 210 \mathrm{I}$ & 34 & $18 / 16$ & $66 \pm 10$ & $8231 \pm 10,257$ & $4652-11,810$ & $10,548 \pm 9985$ & $27 / 7$ & 0.18 & 0.63 \\
\hline $\begin{array}{l}\text { FFI-D178N- } \\
\text { M }\end{array}$ & 26 & $9 / 17$ & $50 \pm 12$ & $2871 \pm 2704$ & $1779-3963$ & $993 \pm 1715$ & $4 / 22$ & 0.22 & 0.19 \\
\hline iCJD & 10 & $5 / 5$ & $50 \pm 11$ & $4709 \pm 2712$ & $3025-6036$ & $12,171 \pm 10,053$ & $8 / 2$ & 0.42 & 0.44 \\
\hline $\mathrm{AD}$ & 35 & $22 / 13$ & $67 \pm 10$ & $2896 \pm 1603$ & $2345-3447$ & $633 \pm 401$ & NA & 0.41 & 0.25 \\
\hline
\end{tabular}

Number of cases $(n)$, age [mean value \pm standard deviation (SD)], sex distribution ( $f$ female, $m$ male), CSF sTREM2 [mean value \pm SD, and $95 \%$ confidence interval $(95 \% \mathrm{CI})]$ and $t$-tau (mean value $\pm \mathrm{SD}$ ) concentrations as well as 14-3-3 positivity (pos/positive, neg/negative) are indicated. Inconclusive 14-3-3 tests were considered as negative. Significant associations ( $p$ value) between sTREM2 with sex and age for each diagnostic group are reported. *For 13 cases, t-tau data were not available. (NA not available) 
criteria [76], genetic CJD $(n=91)$, iatrogenic CJD $(n=10)$, and fatal familial insomnia (FFI, $n=26$ ) were collected in the following CJD reference centres: (1) Clinical Dementia Centre and the National Reference Centre for CJD Surveillance at the University Medical Centre, Göttingen, Germany, (2) Alzheimer's Disease and Other Cognitive Disorders Unit, Hospital Clínic, Barcelona, Spain, (3) National Centre of Microbiology-Carlos III Institute of Health, Madrid, Spain, and (4) Istituto Superiore di Sanità, Rome, Italy. The diagnosis of genetic prion diseases was carried out according to surveillance criteria after prion protein gene (PRNP) analysis [31] and following World Health Organization (WHO) criteria [73]. Iatrogenic CJD was diagnosed according to established WHO criteria [73]. Nine iatrogenic cases were associated with dura mater grafts and one with corneal transplantation. $\mathrm{AD}$ cases were diagnosed according to the National Institute on Aging-Alzheimer's Association workgroups (NIA-AA) criteria [45].

In addition, CSF was obtained from patients with relapsing/remitting multiple sclerosis [69] at the Multiple Sclerosis Unit, Service of Neurology, Bellvitge University Hospital $(n=16)$, and the Clinical Dementia Centre and the National Reference Centre for CJD Surveillance at the University Medical Centre of Göttingen (Germany) ( $n=13$ (Supplementary table 4).

Cerebrospinal fluid tests in healthy controls (HC) was obtained from patients for knee surgical procedures under spinal anaesthesia.

The ND group was composed of cases diagnosed with neurological diseases not associated with neurodegenerative pathology and included the following diagnostic groups: psychosis, paranoid psychosis, bipolar disorder, schizophrenia, ischemic stroke, multiple infarct, epilepsy, meningitis, alcohol abuse, vertigo, acute or chronic headache, pain syndromes, acute hypoxia, polyneuropathy, cerebral lymphoma, astrocytoma, and paraneoplasic syndromes. ND cases were diagnosed according to acknowledged standard neurologic clinical and para-clinical findings based on the International Classification of Diseases 10th Revision definitions.

Blood was collected in Plasma-EDTA tubes at the Department of Transfusion Medicine (healthy controls, HC; $n=97$ cases) and at the Department of Neurology (sCJD cases, $n=86$ cases) of the University Medical Centre Göttingen (Germany) under standardized pre-analytical conditions. The healthy control (HC) group was composed of healthy blood donors without any relevant clinical findings (Table 2).

For the analysis of CSF sTREM2 concentrations at different disease stages in SCJD, we divided the time from lumbar puncture (LP) or blood uptake to disease onset in each patient by the total duration of the disease. Then, samples were grouped into three categories according to whether they underwent LP/blood uptake in the first (time of LP to disease onset/total duration of the disease $<0.33$ ), second (0.33-0.66), or third ( $>0.66)$ stage of the disease, as previously reported [59].

\section{CSF and plasma tests}

Soluble TREM2 (sTREM2) concentrations in the CSF and plasma were quantified with a previously described immunoassay [29] using the electrochemiluminescence-based MesoScale Discovery platform. Inter- and intra-assay coefficients of variation in our study were below $12 \%$ and $10 \%$, respectively. CSF from MS cases was assessed in two series. In the first, the method was the previously described immunoassay [29], while for the second series (Bellvitge) assessment was made using the Human TREM2 ELISA Kit (Abcam, ab224881). CSF total-tau (t-tau) was quantified using a commercially available colorimetric enzyme-linked immunosorbent assay (INNOTEST hTAU-Ag from Fujirebio). CSF was analysed for the presence of 14 to 3-3 protein using western blotting [75]. CSF and plasma YKL-40 were quantified using the MicroVue YKL-40 ELISA kit from Quidel. Plasma neurofilament light (NfL) and t-tau concentrations were measured using commercially available assays on the Single molecule array (SIMOA) HD-1 instrument (Quanterix). The analysts were masked to clinical data.

\section{Animal experimentation}

We conducted animal experiments in accordance with the Code for Methods and Welfare Considerations in Behavioural Research with Animals (Directive 2010/63/EU)
Table 2 Demographic and biomarker characteristics of the cases used in the plasma cohort study

\begin{tabular}{|c|c|c|c|c|c|c|c|}
\hline & \multirow[t]{2}{*}{$n$} & \multirow[t]{2}{*}{$\operatorname{Sex}(f / m)$} & \multirow{2}{*}{$\begin{array}{l}\text { Age } \\
\text { Mean + SD }\end{array}$} & \multirow{2}{*}{$\begin{array}{l}\text { sTREM2 pg/mL) } \\
\text { Mean + SD }\end{array}$} & \multirow[t]{2}{*}{$95 \%$ CI } & \multicolumn{2}{|l|}{$p$ values } \\
\hline & & & & & & sTrem 2 vs. Sex & sTrem 2 vs. age \\
\hline $\mathrm{HC}$ & 97 & $23 / 74$ & $64 \pm 5$ & $4409 \pm 3961$ & $3611-5208$ & 0.58 & 0.12 \\
\hline sCJD & 86 & $52 / 34$ & $65 \pm 8$ & $8981 \pm 8932$ & $7066-10,896$ & 0.94 & 0.11 \\
\hline
\end{tabular}

Diagnostic groups included HC and sCJD. Number of cases $(n)$, age: mean value \pm standard deviation (SD), sex distribution ( $f$ female, $m$ male), and plasma sTREM2 concentrations: mean value \pm SD and $95 \%$ confidence interval (95\% CI) are indicated. Significant associations ( $p$ value) between sTREM2 with sex and age for each diagnostic group are indicated 
and made every effort to minimize suffering. Experiments developed in the Centro de Investigación en Sanidad Animal-Instituto Nacional de Investigación y Tecnología Agraria y Alimentaria (Madrid, Spain) were evaluated by the Committee on the Ethics of Animal Experiments of the Instituto Nacional de Investigación y Tecnología Agraria y Alimentaria and approved by the General Directorate of the Madrid Community Government (permit no. PROEX 094/18). The tg340 and tg361 mouse lines expressing about four-fold level of human PrPM129 and PrPV129 on a mouse PrP null background were used as mouse models of SCJD MM1 and SCJD VV2 subtypes. Individually identified 6-to10-week-old mice were anaesthetized and inoculated with $2 \mathrm{mg}$ of a $10 \%(\mathrm{w} / \mathrm{v}$ ) brain homogenate of SCJD MM1 (in the $\operatorname{tg} 340$ mouse line) and SCJD VV2 (in the tg361) in the right parietal lobe using a 25-gauge disposable hypodermic needle. Control animals for each genotype were inoculated with control brain homogenates. Mice were observed daily and their neurological status was assessed weekly. At 80, 120 , or 180 days post-inoculation (dpi) animals were euthanized, necropsy was performed, and the brain was removed. Four to eight animals per time point and condition were used for RT-qPCR assays.

\section{Western blotting}

Human tissues were lysed in RIPA lysis buffer RIPA $(\mathrm{NaCl}$ $1 \mathrm{M}$, Nonidet P-40 1\%, sodium deoxycholate $0.5 \%$,SDS $0.1 \%$, Tris $50 \mathrm{mM}, \mathrm{pH} 7.4$ ) supplemented with protease, and phosphatase inhibitors (Roche, GE). After centrifugation at $14,000 \mathrm{~g}$ for $20 \mathrm{~min}$ at $4{ }^{\circ} \mathrm{C}$, supernatants were quantified for protein concentration (BCA, Pierce), mixed with SDSPAGE sample buffer, boiled, and subjected to $4-12 \%$ BisTris Gels (NuPAGE ${ }^{\mathrm{TM}} 4$ to $12 \%$, Bis-Tris, $1.5 \mathrm{~mm}$, Mini Protein Gel, 15-well, ThermoFisher NP0336BOX). Gels were transferred onto PVDF membranes and processed for specific immunodetection. Membranes were incubated overnight at $4{ }^{\circ} \mathrm{C}$ with primary antibodies and with appropriate horseradish peroxidase-conjugated secondary antibody (anti-mouse/ anti-rabbit HRP-IgG) diluted at 1:2000 (Dako) for $1 \mathrm{~h}$ at room temperature. Chemiluminescence immunodetection was performed using the ECL-Amersham (GE Healthcare) kit. Densitometry was carried out with the ImageJ software and values were normalized using $\beta$-actin levels.

\section{Real-time polymerase chain reaction (qPCR)}

RNA from different human and mouse brain regions was purified using RNeasy Plus Mini Kit (Qiagen, 74,136), which eliminates genomic DNA contamination. RNA integrity was assessed with the RNA Integrity Number (RIN value) determined with the Agilent 2100 Bioanalyzer (Agilent) (RIN values between 4 and 9.2). Reverse transcription of the RNA samples was carried out using the High-Capacity cDNA Reverse Transcription kit (Applied Biosystems) following the supplier's instructions. Quantitative RT-PCR assays were performed in duplicate on cDNA samples in 384-well optical plates with ABI Prism 7900 Sequence Detection System (Applied Biosystems, Life Technologies). The reactions were carried out using 20xTaqMan Gene Expression Assays and 2xTaqMan Universal PCR Master Mix (Applied Biosystems) with the following probes: CD68 Hs00154355_m1, TREM2 human Hs00219132_m1, TREM2 mouse Mm04209424_g1, and ADAM10 human Hs00153853_m1. The reactions were conducted using the following parameters: $50{ }^{\circ} \mathrm{C}$ for $2 \mathrm{~min}, 95^{\circ} \mathrm{C}$ for $10 \mathrm{~min}$, and 40 cycles with $95{ }^{\circ} \mathrm{C}$ for $15 \mathrm{~s}$ and $60{ }^{\circ} \mathrm{C}$ for $1 \mathrm{~min}$. The fold change was determined by the $\Delta \Delta \mathrm{Ct}$ method, in which each target gene is normalized to its endogenous control to obtain the $\Delta \mathrm{Ct}$. Afterwards, each $\Delta \Delta \mathrm{Ct}$ is determined by subtracting the mean- $\Delta \mathrm{Ct}$ of the control group samples to the $\Delta \mathrm{Ct}$ of each sample. Finally, these $\Delta \Delta \mathrm{Ct}$ values are used as the negative exponent with base 2 to obtain the fold change per sample. Mean fold change values were analysed with appropriate statistical test indicated in each figure using GraphPad Prism 6.01. Gene expression levels were normalized to the housekeeping genes Glucuronidase beta (GUSB) Hs00939627_m1 NM_000181.3 or glyceraldehyde 3-phosphate dehydrogenase (GAPDH) Hs03929097_g1 NM_001289745.1 for human and, GAPDH Mm99999915_ g1 NM_001289726.1 for mouse (Applied Biosystems).

\section{Immunohistochemistry}

De-waxed sections, 4 microns thick, were processed for immunohistochemistry. The sections were boiled in citrate buffer to retrieve antigenicity, followed by endogenous peroxidase blockage (Dako Real peroxidase blocking solution S2023). All sections were incubated overnight at $4{ }^{\circ} \mathrm{C}$ with primary antibodies diluted in Dako Real Antibody Diluent (Dako S2022).

Primary antibody anti-human TREM2 (RD Systems AF1828) diluted 1:20, anti-human TREM2 (Abcam, ab209814) diluted 1:100, and anti-human ADAM10 (Abcam, 1997) diluted 1:150 were used. Detection was achieved by incubation with R.T.U. Biotinylated Universal Antibody (Abcam ab64257) for $10 \mathrm{~min}$ at room temperature, followed by R.T.U. HRP-Streptavidin (Abcam ab64269). The peroxidase reaction was visualized with diaminobenzidine and $\mathrm{H}_{2} \mathrm{O}_{2}$. Control of the immunostaining included omission of the primary antibody.

\section{Immunofluorescence}

De-waxed sections, 4 microns thick, were boiled in citrate buffer to enhance antigenicity, and afterwards were stained 

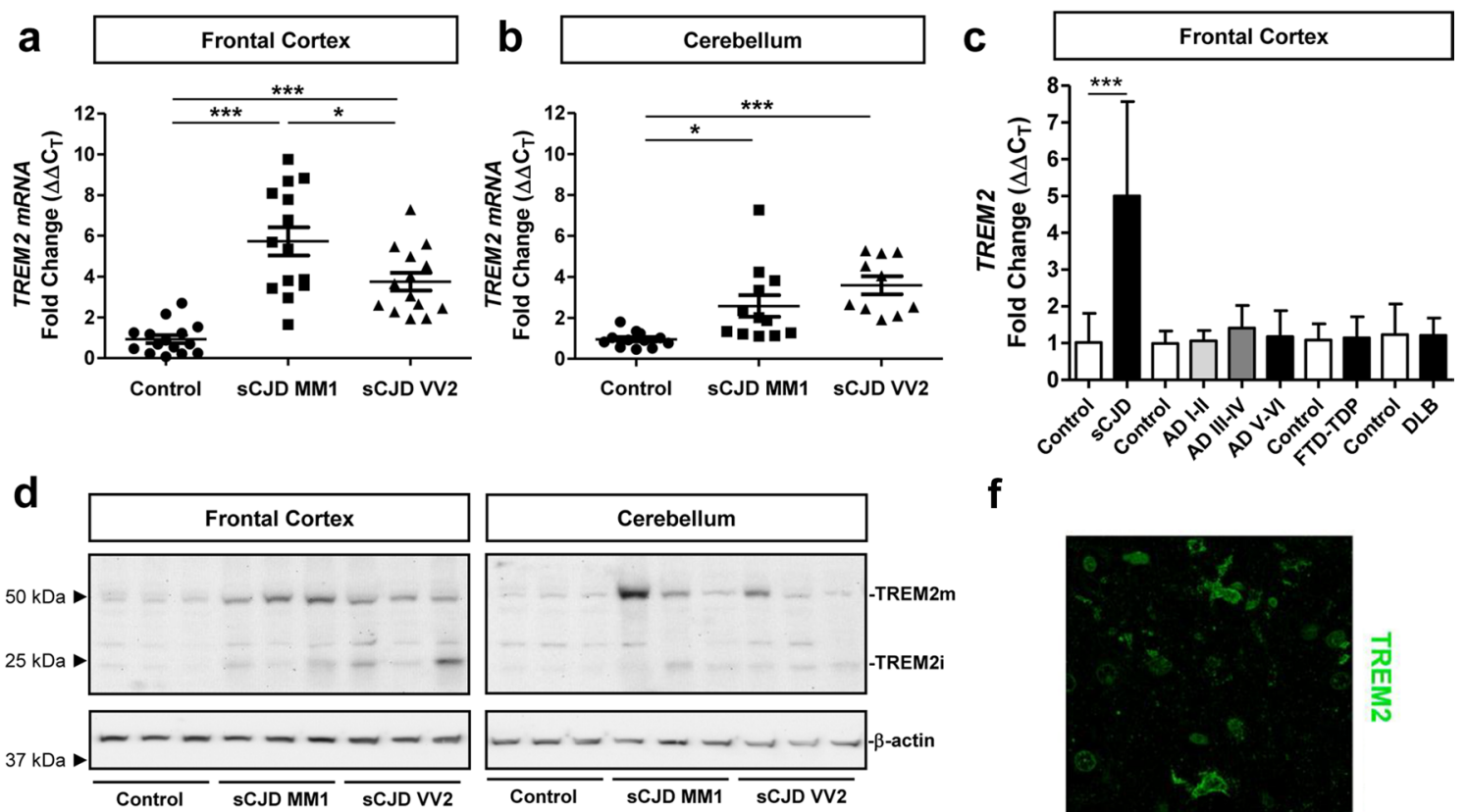

f
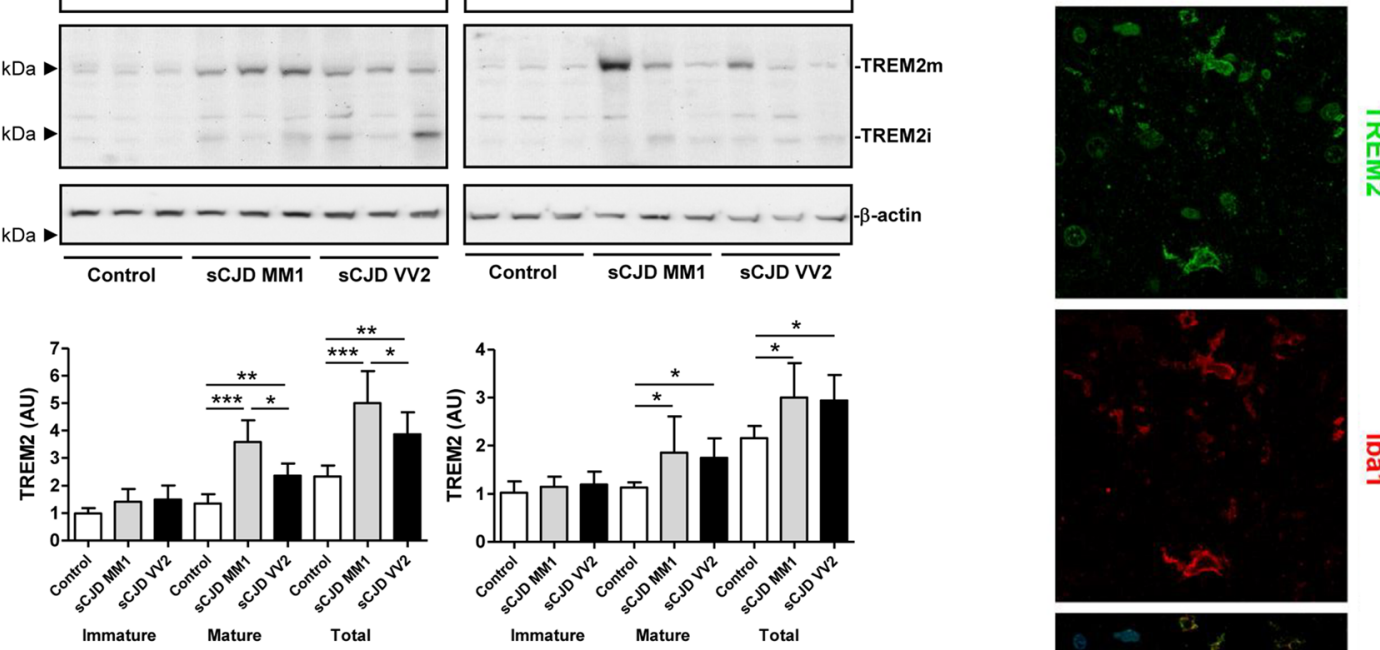

e
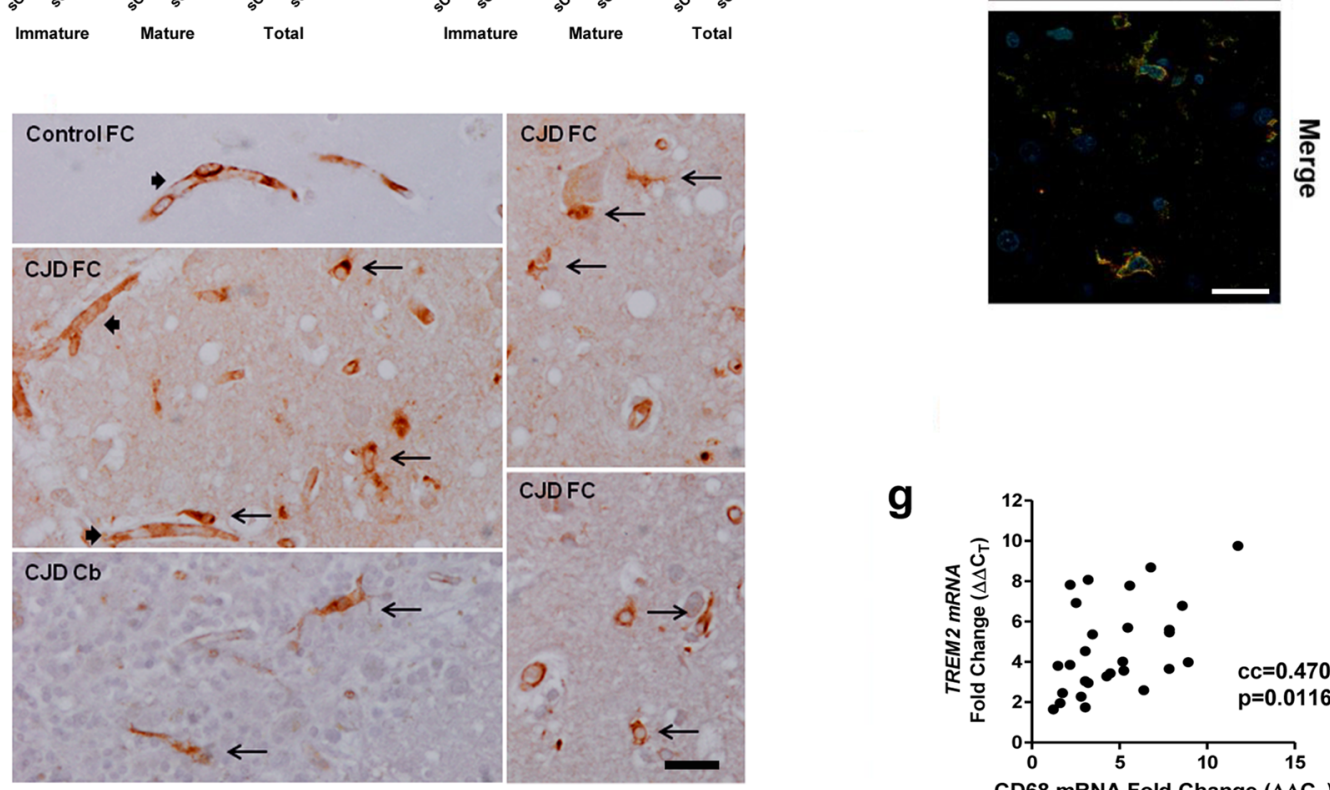

g

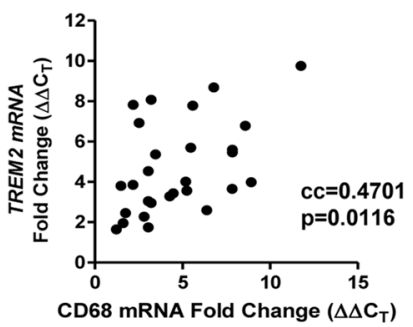

with a saturated solution of Sudan black B (Merck, DE) to block auto-fluorescence of lipofuscin granules present in cell bodies. Then they were rinsed in $70 \%$ ethanol and washed in distilled water. All sections were blocked at room temperature with $10 \%$ foetal bovine serum diluted in PBS, and incubated at $4^{\circ} \mathrm{C}$ overnight with combinations of primary antibodies. After washing, the sections were incubated with Alexa-488 and Alexa-555 (Molecular Probes, Eugene, Oregon, USA) fluorescence secondary antibodies. Nuclei were stained with DRAQ5 ${ }^{\mathrm{TM}}$ (dilution 1:2,000, BioStatus, Loughborough, UK). After washing, 
४Fig. 1 TREM2 expression in sCJD brain tissue. RT-qPCR analysis of TREM2 in: a the frontal cortex (FC) and $\mathbf{b}$ cerebellum (CB) of control, sCJD MM1, and sCJD VV2 cases. GAPDH was used for normalization. FC (controls $n=15$, sCJD MM1; $n=11$, sCJD VV2; $n=11$ ), CB (controls $n=12$, sCJD MM1; $n=12$, sCJD VV2; $n=10$ ). c RTqPCR analysis of TREM2 in the FC of SCJD (MM1 and VV2), and FTD-TDP. For each neurodegenerative dementia, data were compared to age and sex-matched controls. CJD (controls $n=14$, sCJD (MM1 and VV2); $n=22$ ), FTD-TDP-43 (controls $n=17$, FTD-TDP-43; $n=16)$. d Representative western blot analysis of TREM2 in the FC and CB of control, sCJD MM1, and SCJD VV2 cases. TREM2 immature (TREM2i) and mature (TREM2m) forms are indicated. For normalization $\beta$-actin was used. Graphical representation of western blot data acquired from the analysis of FC (controls $n=8$, sCJD MM1; $n=8$, sCJD VV2; $n=10$ ) and CB (controls; $n=8$, sCJD MM1; $n=8$, SCJD VV2; $n=8)$ is shown; $* p<0.05, * * p<0.01, * * * p<0.001$. e TREM-2 immunohistochemistry in frontal cortex and cerebellum in control cases, and then in frontal cortex in CJD MM1. In control and CJD cases, TREM2 immunoreactivity is localized in the blood vessels (thick arrows); in CJD, TREM2 immunoreactivity is also observed in glial cells with the morphology of microglia (long thin arrows); scale bar $=30 \mu \mathrm{m}$. f TREM 2 double-labelling immunofluorescence and confocal microscopy in FC of sCJD showing TREM2 localization in a subset of microglial cells identified with the antibody Iba1 (white arrows). Nuclei are stained with DRAQ5 ${ }^{\mathrm{TM}}$ (blue); scale bar $=20 \mu \mathrm{m}$. $\mathbf{g}$ Correlation analysis between TREM 2 and $C D 68$ mRNA in the FC of SCJD cases $(n=28)$. cc: correlation coefficient

the sections were mounted in Immuno-Fluore mounting medium (ICN Biomedicals, Irvine, CA, USA), sealed, and dried overnight. Sections were examined with a Leica TCS-SL confocal microscopy.

\section{Statistical analysis}

For brain tissue data, in two group comparisons, non-parametric Mann-Whitney- $U$ tests were used. For comparisons between multiple groups, Kruskal-Wallis tests followed by Dunn's post-hoc test or one-way ANOVA followed by Tukey post-hoc test were applied after testing for normality. Biomarker data were log-transformed, and linear models were built controlling for the effect of age and sex. To assess the diagnostic accuracy of sTREM2, receiver operating characteristic (ROC) curve analyses were carried out and areas under the curve (AUC) with confidence intervals (95\% CI) were calculated. Spearman rank correlation coefficients were used to assess associations between continuous biomarker levels. Statistical comparison between ROC curves was made with the pROC R package [57]. Longitudinal analysis of sTREM2 along disease stages was conducted with nlme $\mathrm{R}$ package [53] using a multilevel linear model that assumed a fixed slope for disease stage.

\section{Results}

\section{TREM2 and ADAM10 expression in the brain of SCJD patients}

Expression of TREM2 was analysed in the frontal cortex (FC) and cerebellum (CB) of SCJD MM1 and VV2 cases. With RT-qPCR analysis, TREM2 mRNA was significantly increased in the FC of SCJD MM1 $(n=14, p<0.001$, fold change 6.1) and VV2 $(n=14, p<0.001$, fold change 3.5$)$ cases compared to controls $(n=15)$. TREM2 mRNA expression was higher in sCJD cases possessing the $P R N P$ gene codon $129 \mathrm{MM} 1$ as compared to VV2 genotype $(p<0.05)$ (Fig. 1a). In the CB, TREM2 mRNA was significantly increased in SCJD VV2 $(n=10, p<0.001)$ and MM1 $(n=12$, $p<0.05)$ compared to controls $(n=12)$ (Fig. 1b). TREM2 levels were normalized to $G A P D H$, and similar results were obtained for GUSB normalization (data not shown). Increased TREM2 mRNA expression in the FC in SCJD was significant $(n=28, p<0.001)$ when compared with FTLDTDP ( $n=16)$ (Fig. 1c).

Western blot analysis revealed significantly increased protein levels of mature and total TREM2 (mature plus immature forms) in the FC of SCJD cases compared to controls ( $p<0.001$, control vs. sCJD MM1 and $p<0.01$, control vs. sCJD VV2 for total TREM2) (Fig. 1d). The expression of mature and total TREM2 was significantly higher in SCJD MM1 cases compared to SCJD VV2 $(p<0.05)$. In the CB, mature and total TREM2 was significantly higher in SCJD cases compared to controls ( $p<0.05$ in all comparisons) (Fig. 1d). In both brain regions, 10 controls, 8 sCJD MM1, and 8 sCJD VV2 cases were used.

In immunohistochemistry study of control brains using the TREM2 polyclonal RD Systems, AF1828H antibody disclosed immunostaining in the blood vessel walls, with no immunostaining of neurons or glial cells (data not shown). Using the same antibody, TREM2 immunoreactivity in sCJD MM1 brains was observed in the blood vessel walls and in microglia; neurons were not immunostained with this antibody (Fig. 1e). De-waxed paraffin sections of AD, DLB, MS, sALS, and VD cases showed TREM2 immunoreactivity in the blood vessels and microglia surrounding $\beta$-amyloid plaques in AD, lipid-laden macrophages in subacute MS plaques, macrophages and a few mononuclear cells in subacute infarcts, and a few microglia in the spinal cord in sALS. Neurons were not stained with this antibody (data not shown). To validate immunohistochemical data, doublelabelling immunofluorescence against TREM2 and Iba- 1 in the FC of sCJD MM1 cases disclosed TREM2 localization in a subpopulation of microglial cells as revealed with the IBA1 antibody (Fig. 1f). Additionally, TREM2 mRNA in 
FC of SCJD cases correlated positively with the macrophage marker CD68 ( $n=28, \mathrm{cc}=0.4701, p=0.0116)$ (Fig. 1g).

In contrast, immunohistochemistry with the anti-TREM2 Abcam antibody showed neuronal immunoreactivity in control and diseased cases, in addition to immunostaining of the blood vessel walls and rare microglia (data not shown). We tried to reproduce these observations using another batch but the TREM2 Abcam antibody is a discontinued product and no longer available.

Next, we explored whether ADAM10, the main TREM2 sheddase responsible for its regulated intramembrane proteolysis [60], was also altered in SCJD patients. With RTqPCR analysis, no alterations in ADAM10 mRNA levels were observed in SCJD, either in the FC (controls, $n=15$;
Fig. 2 ADAM10 expression in sCJD brain tissue. a RT-qPCR analysis of $A D A M 10$ in the frontal cortex (FC) and cerebellum (CB) of control, sCJD MM1, and SCJD VV2 cases. GAPDH was used for normalization. FC (controls $n=15$, sCJD MM1; $n=11, \operatorname{sCJD}$ VV2 $n=10), \mathrm{CB}$ (controls $n=12$, sCJD MM1; $n=12, \operatorname{sCJD}$ VV2 $n=10)$. b

Representative western blot analysis of ADAM10 in the FC and $\mathrm{CB}$ of control, sCJD MM1, and SCJD VV2 cases. ADAM10 precursor (ADAM10p) and mature (ADAM10m) forms are indicated. $\beta$-actin was used for normalization. Graphical representation of western blot data acquired from the analysis of FC (controls $n=12$, sCJD MM1; $n=12$, sCJD VV2; $n=12$ ) and CB (controls; $n=9$, SCJD MM1; $n=10$, sCJD VV2; $n=10) ; * p<0.05, * * p<0.01$, $* * * p<0.001$. c ADAM10 immunohistochemistry in the frontal cortex (FC), cerebellum $(\mathrm{Cb})$, and subcortical white matter (WM) in control and SCJD MM1. ADAM10 immunoreactivity is observed in neurons in the FC and granule cells (gr) of cerebellum, but Purkinje cells (P) are very weakly stained or not at all. No positive cells are distinguished in the white matter; scale bar $=20 \mu \mathrm{m}$ a
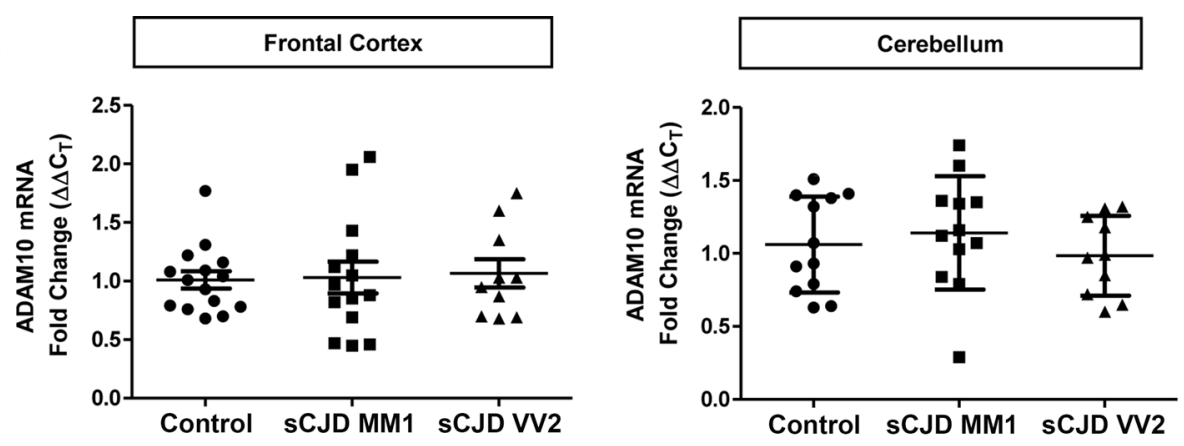

b
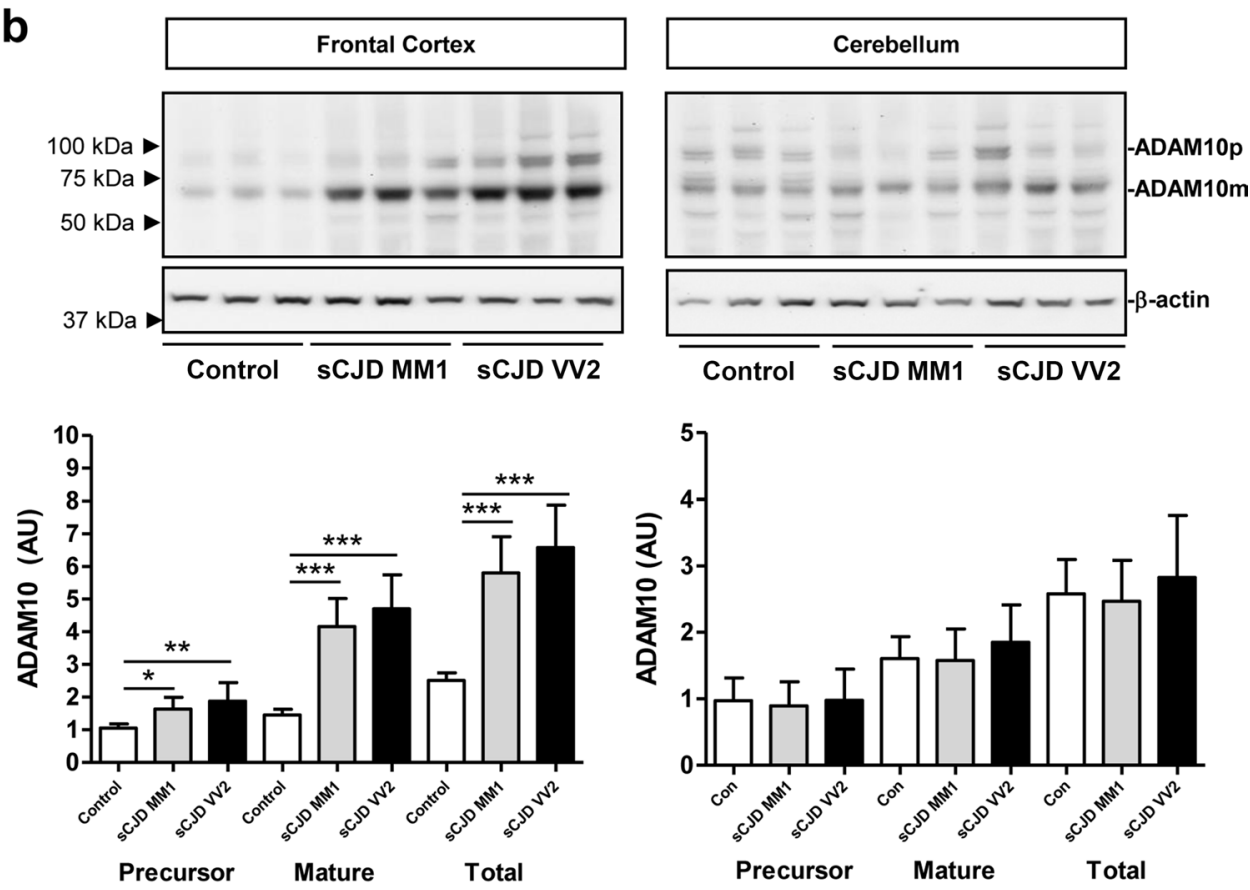

C

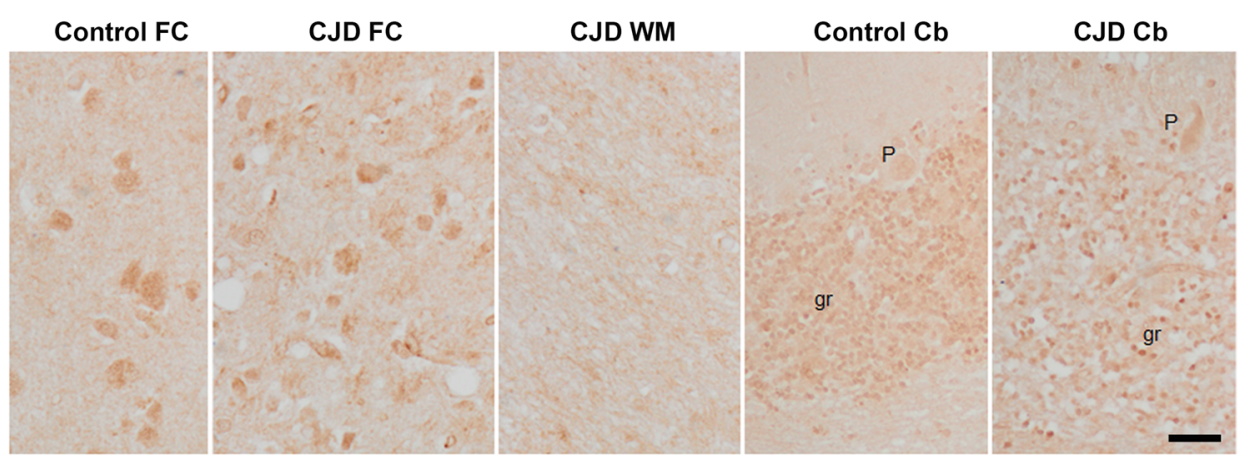


sCJD MM1, $n=14$ and sCJD VV2, $n=10$ ) or in the CB (controls, $n=12$; sCJD MM1, $n=12$ and sCJD VV2, $n=10$ ) (Fig. 2a). TREM2 levels were normalized to GAPDH, and similar results were obtained for GUSB normalization (data not shown). In contrast, ADAM10 precursor and mature forms were increased in western blot in the FC of sCJD MM1 ( $p<0.05$ precursor, $p<0.001$ mature) and sCJD VV2 ( $p<0.01$ precursor, $p<0.001$ mature) compared to controls (Fig. 2b). In the CB, ADAM10 levels were not significantly different for SCJD compared to controls ( $p>0.05$ in all comparisons) (Fig. 2b). In both brain regions, 10 controls, 8 sCJD MM1, and 8 sCJD VV2 cases were used. The ADAM10 antibody showed suboptimal results for immunohistochemistry; ADAM10 immunostaining was weak and blurred. ADAM10 immunoreactivity was moderate in neurons of the FC, weaker in the granular cells of the cerebellum and negative in Purkinje cells, in control and SCJD MM1 cases (Fig. 2c). sCJD VV2 was not analysed. Incubation with only the secondary antibody showed no immunoreactions (data not shown).

\section{Regional and temporal TREM2 expression in the brain of SCJD mouse models}

To investigate the regional and temporal alterations of TREM2 during prion pathogenesis in brain tissue, we took advantage of two sCJD mouse models recapitulating the molecular and pathological alterations of sCJD MM1 (tg340-sCJD MM1) and sCJD VV2 (tg360-sCJD VV2) subtypes [9, 10, 34]. Trem2 mRNA expression was investigated in the anterior and posterior cortex, cerebellum, olfactory bulb, and brainstem at pre-clinical (80 dpi), early clinical (120 dpi), and clinical (180 dpi) disease stages $(n=4-8)$ (Fig. 3). At the clinical stage, Trem 2 mRNA was increased in all brain regions in both CJD models. Increased Trem 2 expression was also observed at early-clinical stages in the anterior cortex of both models. Differences between sCJD models were detected in the posterior cortex, where Trem 2 was specifically increased at preclinical and early-clinical stages in the tg340 sCJD model and in the cerebellum, where Trem 2 mRNA expression was specifically increased at earlyclinical stages in the tg361 sCJD model (Fig. 3).

\section{STREM2 in the CSF of prion diseases}

The presence of increased TREM2 expression and ADAM10 expression and activation in the brain of $\mathrm{SCJD}$ raised the possibility that STREM 2 could be differentially altered in biological fluids of SCJD cases. CSF TREM2 concentrations were assessed in $\mathrm{HC}$, ND, prion diseases from sporadic, genetic and acquired aetiology, AD, and MS (Table 1). sTREM2 was associated neither with age at onset nor with sex (Table 1), both when all cases were analysed together and when stratified between non-prion and prion diseases.

Compared to $\mathrm{HC}$ and ND, sTREM2 concentrations were significantly higher in all types of prion diseases, with the exception of FFI cases (Fig. 4a). sTREM2 mean concentrations were higher in ND $(2413 \pm 1262 \mathrm{pg} / \mathrm{mL})$ than in $\mathrm{HC}$ $(1901 \pm 878 \mathrm{pg} / \mathrm{mL})$. Accordingly, the AUC derived from the sCJD vs. HC comparison (0.90) was higher than that for the sCJD vs. ND comparison (0.85) (Fig. 4b). An optimal cutoff of $2650 \mathrm{pg} / \mathrm{mL}$ sTREM2 discriminated HC from sCJD cases with $79 \%$ sensitivity and $94 \%$ specificity, while a cutoff of $2850 \mathrm{pg} / \mathrm{mL}$ sTREM 2 discriminated ND from sCJD cases with $77 \%$ sensitivity and $80 \%$ specificity. AUCs for the discrimination of gCJD E200K, gCJD V210I, and iCJD from $\mathrm{HC}$ and ND showed a good diagnostic accuracy ranging from 0.78 to 0.90 (compared to $\mathrm{HC}$ ) and from 0.73 to 0.85 (compared to ND). In contrast, sTREM2 showed no diagnostic value in distinguishing $\mathrm{HC}$ and ND from FFI cases (AUC 0.62 and 0.51, respectively) (Fig. 4b). sTREM2 was also quantified in AD cases. Mean sTREM2 concentrations in AD were slightly higher than in HC and ND; however, differences did not reach statistical significance (Table 1 and Fig. 4a). In contrast, sTREM2 levels were significantly higher in sCJD and gCJD-V210I compared to AD (Fig. 4a). sTREM2 levels were also assessed in two series of MS cases using different populations and different methods. sTREM2 levels in the CSF were not altered in MS when compared with controls (data not shown).

Next, we explored the influence of codon 129 polymorphism in PRNP on sTREM2 levels. The highest sTREM2 concentrations were detected in $\mathrm{SCJD}$ cases harbouring methionine/methionine (MM; $7884 \pm 6652 \mathrm{pg} / \mathrm{mL}, n=32$ ), followed by methionine/valine (MV; $5257 \pm 4061 \mathrm{pg} / \mathrm{mL}$, $n=19$ ) and valine/valine (VV; $4183 \pm 3803 \mathrm{pg} / \mathrm{mL}, n=17$ ). Statistical differences were detected between MM and VV cases $(p=0.03)$ (Fig. 5a). pROC analysis revealed a superior CSF sTREM2 diagnostic accuracy for sCJD MM compared to sCJD VV cases, both when compared to HC $(\mathrm{AUC}=0.94$ vs $0.82, p=0.0177)$ and to $\mathrm{ND}(\mathrm{AUC}=0.90$ vs. $0.74, p=0.0076$ ) (Fig. 5a).

We further evaluated CSF sTREM2 in the two most prevalent sCJD subtypes (MM1 and VV2) in those cases with prion type available from autopsy analysis. sCJD MM1 $(8196 \pm 7782 \mathrm{pg} / \mathrm{mL}, n=20)$ presented higher sTREM2 concentrations than sCJD VV2 cases $(3224 \pm 2200 \mathrm{pg} / \mathrm{mL}$, $n=9$ ) (Fig. 5b). In line with these observations, AUC values were significantly different between the two subtypes when compared to $\mathrm{HC}$ ( $\mathrm{AUC}=0.95$ vs $0.81, p=0.0151)$ and $\mathrm{ND}(\mathrm{AUC}=0.90$ vs. $0.73, p=0.0070)$ (Fig. 5b), indicating a significantly higher diagnostic value of sTREM2 in the discrimination of SCJD MM1 cases. Regarding genetic prion diseases, gCJD-E200K and FFI cases were stratified according to their PRNP codon 129 genotype, which influences 
Fig. 3 mRNA Trem 2 expression in the brain of sCJD MM1 and VV2 mouse models. RT-qPCR analysis Trem 2 expression in mouse models of sCJD MM1 (tg340-CJD MM1) (left panels) and sCJD VV2 (tg361-CJD VV2) (right panels) subtypes. Trem2 expression was analysed in five brain regions: anterior and posterior cortex, cerebellum, olfactory bulb, and brainstem, at three time points: pre-clinical (80 days post inoculation (dpi)), early-clinical (120 dpi), and clinical (180 dpi). CJD-inoculated animals (black columns) were compared to their corresponding controlinoculated animals (white columns). Four to eight animals per time point and condition were used. Normalization was performed using the housekeeping gene Hprt; ${ }^{*} p<0.05$, $* * p<0.01$ g340 - sCJD MM1
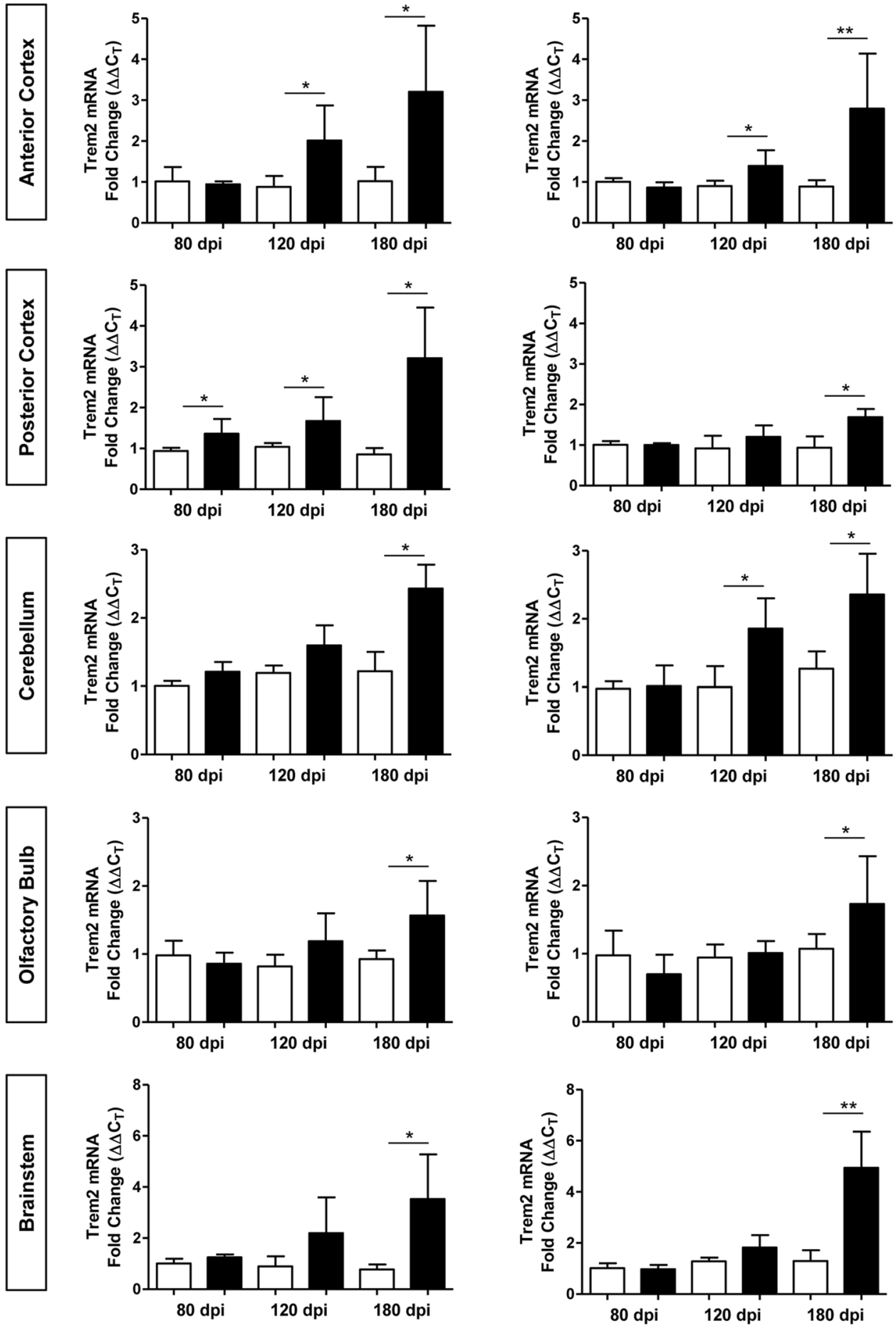

$\square$ Control

sCJD their clinicopathological features [17, 47]. sTREM2 concentrations were not different between gCJD-E200K-MM $(n=33)$ and gCJD-E200K-MV $(n=22)$ cases $(p=0.59)$ nor between FFI-MM $(n=16)$ and FFI-MV $(n=10)$ cases $(p=0.61)$ (data not shown).

Cerebrospinal fluid tests sTREM2 in SCJD displayed a positive correlation with $t$-tau $(n=126, \mathrm{cc}=0.3424$, 
Fig. 4 CSF sTREM2 in the diagnosis of prion diseases. a CSF sTREM 2 concentrations in $\mathrm{HC}(n=48), \mathrm{ND}(n=64)$, $\operatorname{sCJD}(n=139)$, gCJD-E220K $(n=57)$, gCJD-V210 $(n=34)$, FFI $(n=26), \mathrm{iCJD}(n=10)$, and $\mathrm{AD}(n=35)$. b Area under the curve (AUC) derived from receiver operating characteristic (ROC) curves with 95\% confidence interval $(95 \% \mathrm{CI})$ for CSF sTREM2 in the discrimination of prion diseases and $\mathrm{AD}$ from $\mathrm{HC}$ and $\mathrm{ND} ; * p<0.05$, $* * * p<0.001$

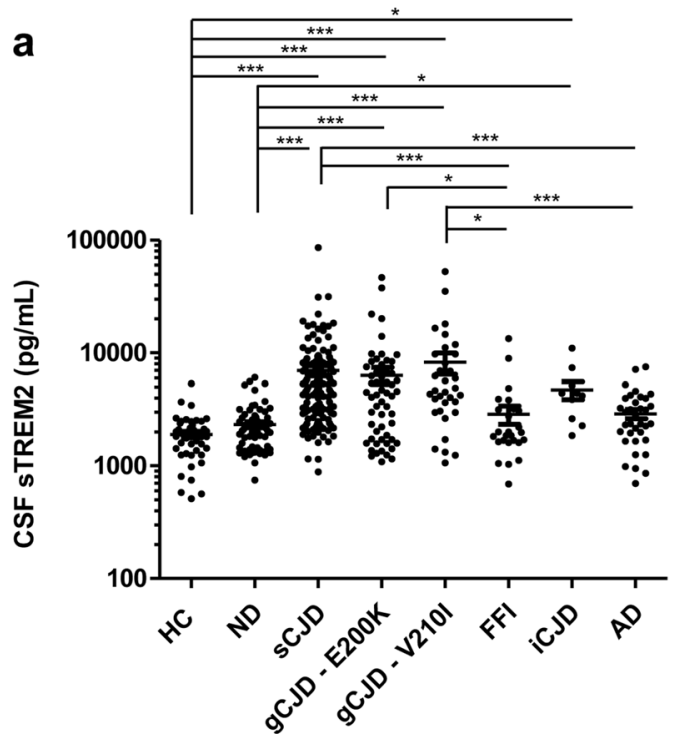

b

\begin{tabular}{|c|c|c|}
\cline { 2 - 3 } \multicolumn{1}{c|}{} & \multicolumn{2}{c|}{ AUC (95\% CI) } \\
\cline { 2 - 3 } \multicolumn{1}{c|}{ HC } & ND \\
\hline gCJD - E200K & $0.78(0.69-0.86)$ & $0.73(0.63-0.81)$ \\
\hline gCJD - V210I & $0.87(0.78-0.97)$ & $0.83(0.72-0.92)$ \\
\hline FFI & $0.61(0.51-0.72)$ & $0.51(0.37-0.64)$ \\
\hline ICJD & $0.90(0.81-0.99)$ & $0.83(0.70-0.96)$ \\
\hline AD & $0.70(0.58-0.82)$ & $0.60(0.48-0.73)$ \\
\hline
\end{tabular}

$p<0.001$ ) (Fig. 5c), and was associated with 14-3-3 positivity, since SCJD cases presenting a positive 14-3-3 test $(n=118)$ showed higher sTREM2 concentrations than those with a negative (or inconclusive) 14-3-3 test ( $n=21$ ) $(p=0.03)$ (Fig. 5d). Additionally, sTREM2 correlated with YKL-40 concentrations $(n=47, \mathrm{cc}=0.3240, p=0.0263)$ (Fig. 5e).

To investigate a potential influence of the disease stage at which the LP was performed and sTREM2 concentrations in sCJD patients, samples were stratified in early $(7914 \pm 5924 \mathrm{pg} / \mathrm{mL}, n=9)$, middle $(8229 \pm 7854 \mathrm{pg} / \mathrm{mL}$, $n=20)$, and late $(6794 \pm 4950 \mathrm{pg} / \mathrm{mL}, n=35)$ stages, but no significant differences were detected between groups (Fig. 5f). In 14 individuals, serial LPs at different disease stages were available allowing investigation of whether sTREM2 values increased during disease progression (Fig. 5g). Using a multilevel linear model, we observed a significant increase of $1962 \mathrm{pg} / \mathrm{mL}$ (95\% CI 811-3113) per disease stage $(p=0.0036)$. CSF sTREM 2 concentrations were not significantly associated with total disease duration $(\mathrm{cc}=-0.1040, p=0.2408)$ (Fig. 5h), ruling out a potential role of CSF sTREM2 as SCJD prognostic marker.

\section{STREM2 in the plasma of SCJD}

Soluble TREM2 concentrations in plasma have been reported to be in the same range as detected in the CSF [52]. Thus, we explored whether sTREM2 in plasma could be altered in SCJD compared to HC. Plasma sTREM2 in $\mathrm{HC}$ and SCJD was associated neither with age at onset nor with sex (Table 2). In sCJD, sTREM2 $(8981 \pm 8932 \mathrm{pg} / \mathrm{mL})$ was significantly increased compared to HC $(4409 \pm 3961)$ $(p<0.001)$ (Table 2, Fig. 6a), with an AUC value of 0.80 in the discrimination of both diagnostic groups (Fig. 6b).

STREM2 concentrations in SCJD were associated neither with PRNP codon 129 genotype: MM $(9755 \pm 10,492 \mathrm{pg} /$ $\mathrm{mL}, n=59)$, MV $(8238 \pm 4220 \mathrm{pg} / \mathrm{mL}, n=8)$ and $\mathrm{VV}$ $(7787 \pm 2773 \mathrm{pg} / \mathrm{mL}, n=14)(p>0.05$ in all comparisons $)$ (Fig. 6c) nor with sCJD subtype: MM1 $(10,040 \pm 11,570$, $n=45)$ and VV2 cases $(7106 \pm 2147, n=12)(p=0.8654)$ (Fig. 6d). In a subset of sCJD cases where plasma-CSF paired data were available $(n=31)$, sTREM2 showed a significant correlation between the fluids ( $\mathrm{cc}=0.3671$, $p=0.0422$ ) (Fig. 6e). Plasma sTREM2 showed a modest positive correlation with plasma YKL-40 $(n=63$, $\mathrm{cc}=0.2958, p=0.0217)($ Fig. 6f) $\mathrm{Nfl}(n=40, \mathrm{cc}: 0.4719$, $p=0.0021)$ (Fig. 6g) and $t$-tau $(n=40, \mathrm{cc}=0.3392$, $p=0.0322$ ) (Fig. 6h) concentrations.

No significant differences in plasma sTREM2 were detected between the different disease stages at which blood was obtained: early $(8258 \pm 3842 \mathrm{pg} / \mathrm{mL}, n=14)$, middle $(7852 \pm 5371 \mathrm{pg} / \mathrm{mL}, n=15)$, and late $(9768 \pm 10,699 \mathrm{pg} /$ $\mathrm{mL}, n=53)$ stages ( $p>0.05$ in all comparisons) (Fig. 6i).

Similar to CSF sTREM2, plasma sTREM2 was not associated with sCJD disease duration $(\mathrm{cc}=-0.1053$, $p=0.3463)$ (Fig. 6j).

\section{Discussion}

In the present study, we report TREM2 expression and levels in the brain and biological fluids of prion diseases, with special focus on SCJD, uncovering its potential role as a diagnostic marker. In SCJD, we observed a significant increase in TREM 2 mRNA compared with controls and 
a

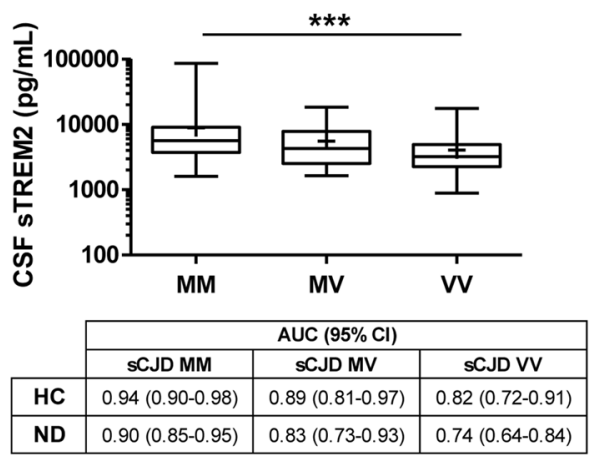

C

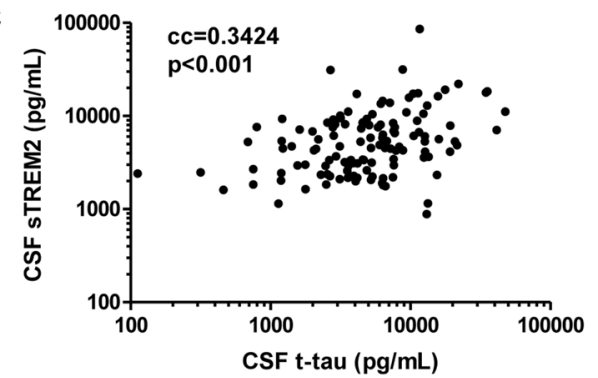

e

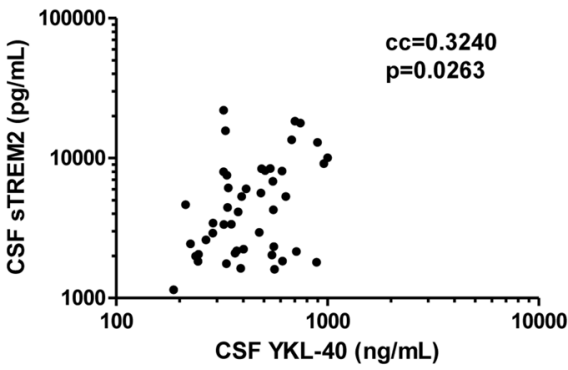

g

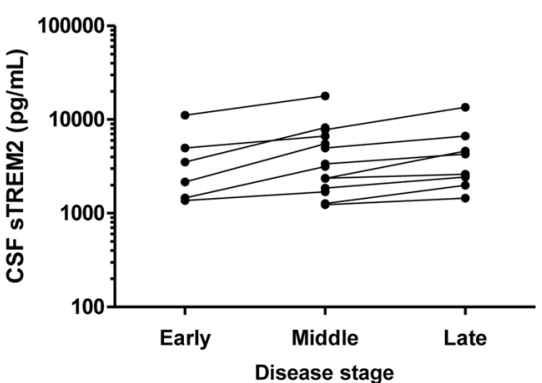

b

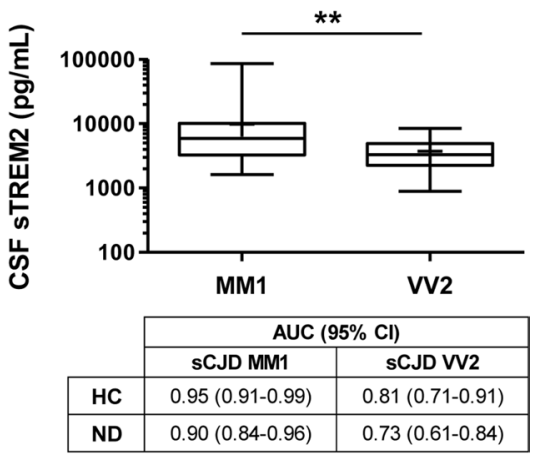

d
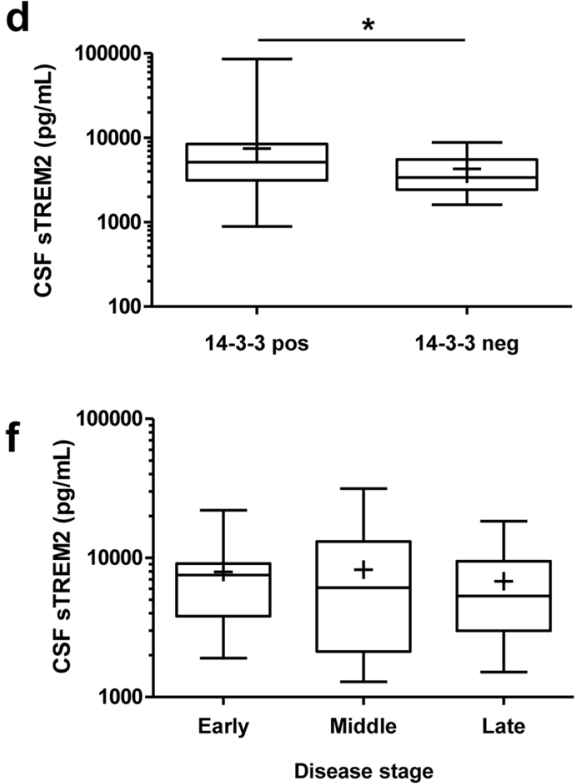

h

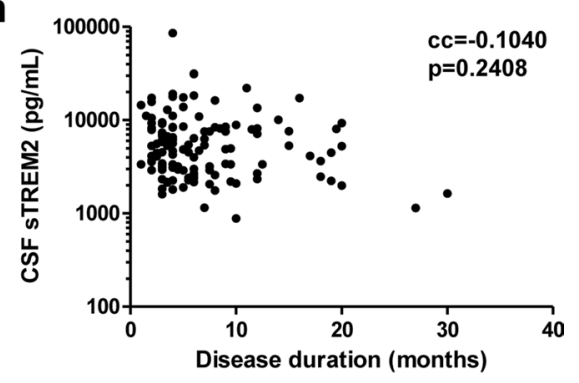

ity. 14-3-3 positive cases; $n=118$ and 14-3-3 negative cases; $n=21$. Mean (+), median (horizontal bar inside the box), and interquartile ranges are represented. e Correlation analysis between CSF sTREM2 and CSF YKL-40 $(n=47)$. f CSF sTREM2 concentrations stratified by disease stage at the time of lumbar puncture (LP). Samples were grouped into three categories according to whether they underwent LP in the first $(<0.33)$, second $(0.33-0.66)$ or third $(>0.66)$ stage of the disease. Early disease stage, $n=15$; middle disease stage, $n=21$; late disease stage, $n=60$. Mean $(+)$, median (horizontal bar inside the box), and interquartile ranges are represented. g Longitudinal analysis of CSF TREM2 concentrations in cases with available LP at different disease stages $(n=14)$. h Correlation analysis between sTREM2 concentrations and disease duration (months) $(n=126)$. cc: correlation coefficient; $* p<0.05, * * p<0.01, * * * p<0.001$ 
Fig. 6 Plasma sTREM2

in SCJD: association with codon129 genotype, sCJD subtype, plasma biomarkers, and clinical data. a Plasma sTREM2 concentrations in $\mathrm{HC}(n=97)$ and SCJD $(n=86)$ cases. b Area under the curve (AUC) derived from receiver operating characteristic (ROC) curves with 95\% confidence interval $(95 \%$ CI) for plasma sTREM2 in the discrimination of SCJD from HC. c Plasma sTREM2 concentrations stratified by prion protein gene $(P R N P)$ codon 129 polymorphism $(\mathrm{M}=$ methionine, $\mathrm{V}=$ valine). $\mathrm{sCJD} \mathrm{MM} ; n=59$, SCJD MV; $n=8$ and SCJD $\mathrm{VV} ; \mathrm{n}=14$. Mean $(+)$, median (horizontal bar inside the box), and interquartile ranges are represented. d Plasma sTREM2 concentrations stratified by sCJD subtype MM1 $(n=45)$ and VV2 $(n=12)$. Mean $(+)$, median (horizontal bar inside the box) and interquartile ranges are represented. e Correlation analysis between plasma and CSF sTREM2 $(n=31)$. Correlation analysis between plasma sTREM2 and plasma YKL-40 f $(n=63)$, plasma $\mathrm{Nfl}(n=40)$ g and plasma $t$-tau $(n=40) \mathbf{h}$. $\mathbf{i}$ Plasma sTREM2 concentrations stratified by disease stage at the time of blood uptake. Samples were grouped into three categories according to whether they underwent blood uptake in the first $(<0.33)$, second $(0.33-$ $0.66)$, or third ( $>0.66)$ stage of the disease. Early disease stage, $n=14$; middle disease stage, $n=15$; late disease stage, $n=53$. Mean (+), median (horizontal bar inside the box), and interquartile ranges are represented. j Correlation analysis between plasma sTREM2 concentrations and disease duration (months) $(n=82)$. cc: correlation coefficient; $* * * p<0.001$ (a)

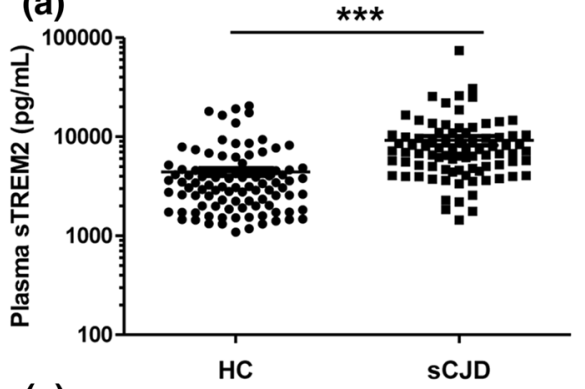

(c)

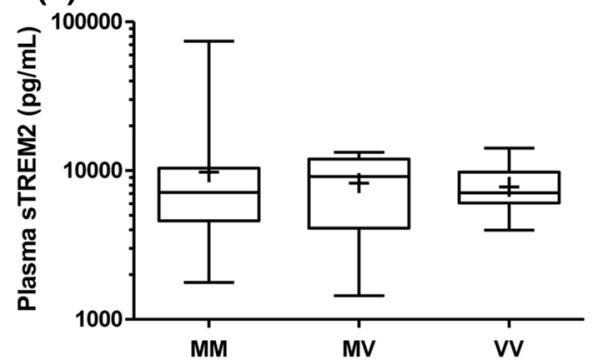

(e)

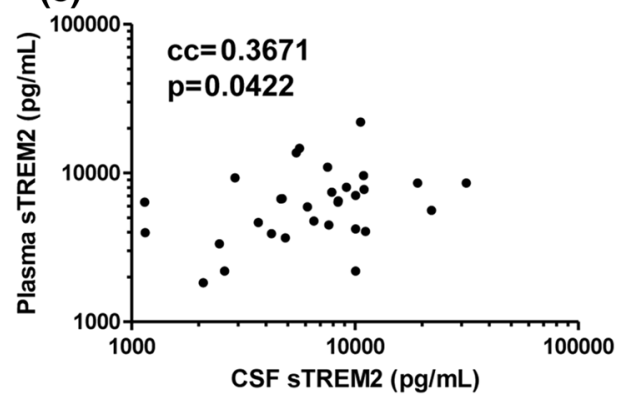

(g)

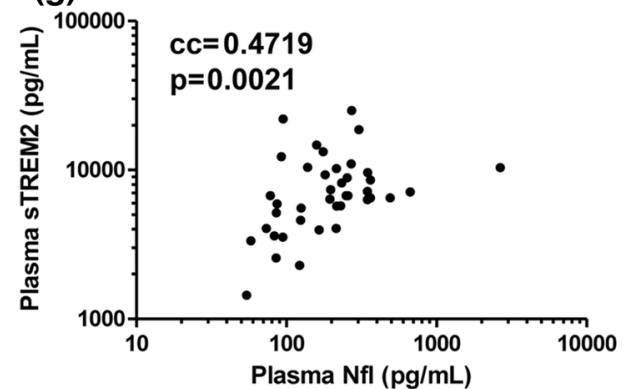

(i)

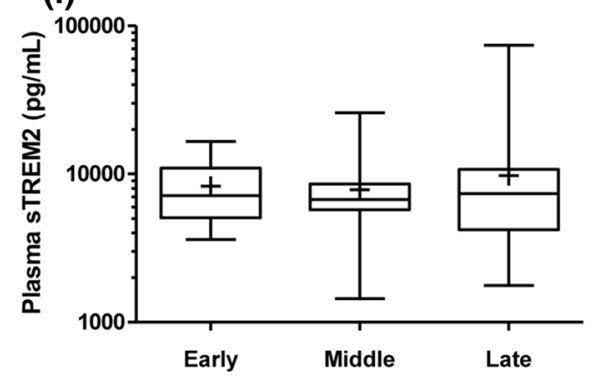

(b)
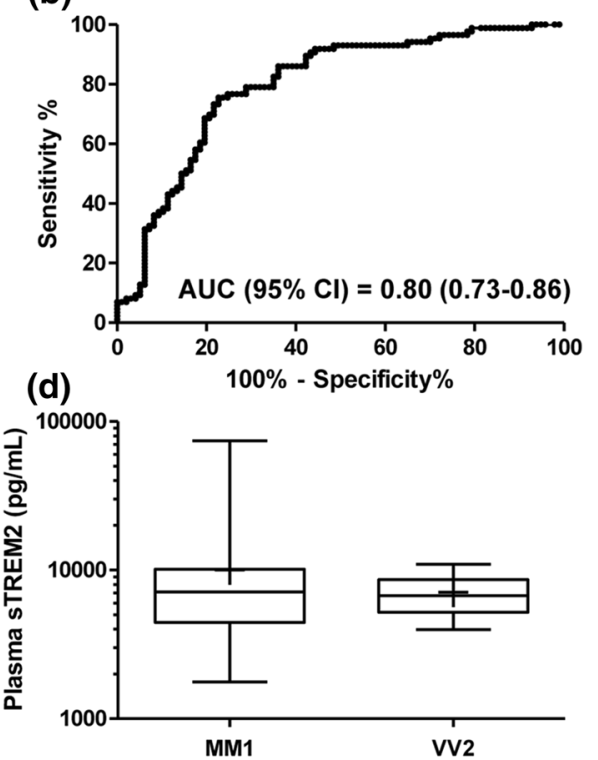

(f)

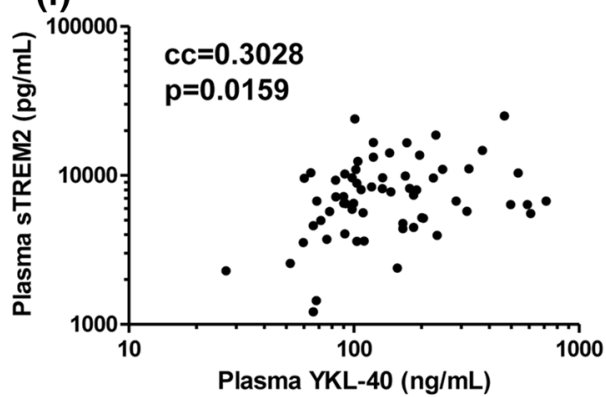

(h)
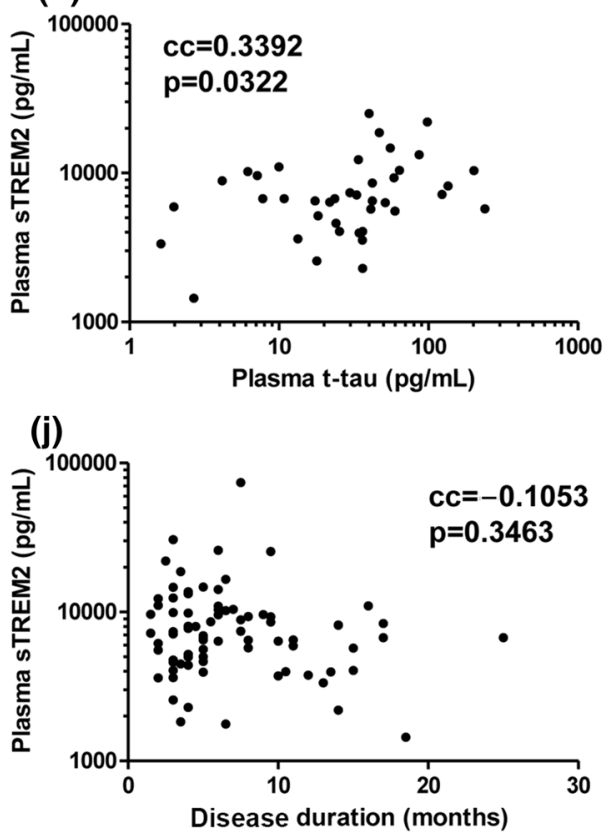
FTLD-TDP43. TREM2 protein is increased in the FC and $\mathrm{CB}$ of SCJD post-mortem-human brain tissue at the protein level (both in its immature and mature forms), as revealed with western blotting. Immunohistochemistry using the anti-TREM2 antibody from RD Systems (AF1828) shows TREM2 expression in blood vessels and microglia. Neurons are also stained using goat anti-TREM2 Abcam antibody in our cases. TREM 2 immunoreactivity has been reported in neurons, astrocytes, and oligodendrocytes using goat anti-TREM2 Abcam antibody in another study [11]. Since there is no evidence of neuronal TREM2 mRNA production in neurons, and the Abcam antibody is discontinued, we assume that additional studies are needed to postulate TREM2 as a neuronal marker.

The positive correlation between TREM2 and the macrophage marker CD68 at the mRNA level supports immunohistochemical findings and suggests that microglia account for the increased TREM2 expression in SCJD brain. This observation, in conjunction with increased ADAM10 expression and activation, points to a potential increase of sTREM2 in biological fluids of prion diseased patients.

In agreement with this hypothesis, sTREM2 displays good accuracy in discriminating SCJD from ND $(\mathrm{AUC}=0.85)$ and $\mathrm{HC}(\mathrm{AUC}=0.90)$ in $\mathrm{CSF}$, and from HC $(\mathrm{AUC}=0.80)$ in plasma. This despite diagnostic accuracies being lower than those reported for CSF biomarkers currently used in clinical settings (14-3-3, $t$-tau ( $p$-tau/t-tau) and RT-QuIC) [13, 36, 44, 62]. The CSF sTREM2 increase in gCJD and iCJD is in line with the similar clinicopathological features of both conditions with SCJD, and consequently, with their fluid biomarkers profiles [32, 38]. In contrast, CSF sTREM2 is not altered in FFI, in agreement with the low sensitivity of prion disease biomarkers in this condition [40], most likely reflecting the restricted pathological alterations in terms of neuroinflammation and longer disease duration compared with CJD cases [8].

Previous studies have shown slightly or moderately increased sTREM2 levels in the CSF in patients with AD [22, 24, 55, 67, 68]. Moreover, individuals with MS and other inflammatory CNS diseases show increased CSF sTREM levels [1, 48, 78]. However, we have not found significant differences in the CSF levels of sTREM2 in AD and MS cases in our series. We can interpret these discrepancies due to lower discrimination capacity of our methods when compared with those used in other labs. If this hypothesis is true, the present findings reinforce the magnitude of sTREM alterations in prion diseases when compared with $\mathrm{AD}$ and MS.

Interestingly, and in contrast to TREM2, SCJD neuroaxonal damage markers with exclusive neuronal expression such as $t$-tau and 14-3-3 display reduced protein levels in sCJD brain tissue [39]. CSF sTREM2 correlates with the neuronal damage markers $t$-tau and 14-3-3 and with the astrocytic marker YKL-40. Similarly, plasma sTREM2 positively correlates with YKL-40, $t$-tau, and Nfl. The moderate but significantly positive association between CSF and plasma sTREM2 in SCJD cases, similar to what is observed in AD [4], suggests that plasma sTREM2 may reflect the CNS neuroinflammatory profile in these conditions. However, the reported expression of TREM2 in non-CNS tissues $[14,74]$, especially in pathological conditions, does not rule out a potential contribution of these tissues to plasma STREM2 concentrations and could explain the differing influence of PRNP codon 129 and SCJD subtype between CSF and plasma.

The influence of PRNP codon 129 on CSF sTREM2 (higher concentrations in MM compared to VV cases) is different to that reported for neuronal damage markers such as CSF t-tau and 14-3-3, which display significantly lower values in CJD MV cases compared to homozygotes [19, 27], and is explained by the less severe phenotype and longer disease duration of CJD MV cases.

Importantly, the increased CSF TREM2 concentrations in sCJD MM compared to SCJD VV cases led to significantly better AUC values in MM cases, reaching high discriminatory power compared to controls $(\mathrm{HC}, \mathrm{AUC}=0.94$ and ND, AUC $=0.90$ ). Thus, data on $P R N P$ polymorphism (routine test in prion diagnostic centres) may improve the accuracy of CSF sTREM2 in terms of disease diagnosis and should be taken into consideration in patient stratification when used as a potential surrogate endpoint in disease-modifying therapy.

The reason for increased CSF sTREM2 in SCJD MM1 compared to SCJD VV2 remains unknown, but it could be explained by the higher microglial profile in the cortical regions of sCJD MM1 compared with VV2 cases [34]. This, together with the observation that increased TREM2 expression is more significant in $\mathrm{FC}$ than in $\mathrm{CB}$, suggests that sTREM2 derived from cortical regions is the main contributor to its increased concentrations in biological fluids.

Another interesting finding in our study is the observation that SCJD mouse models have increased TREM2 levels, occurring in some brain regions at pre-clinical and early-clinical stages in a regional and disease subtype-specific manner. This is in line with the observation that CSF sTREM2 concentrations are sCJD-subtype-specific and independent of the disease stage at which sampling is performed. It is therefore tempting to speculate that sTREM2 alterations in biological fluids may occur at early disease stages. Unfortunately, the absence of a prodromal stage of sCJD precludes the study of pre-clinical cases and leaves this possibility open for the analysis of sTREM2 in asymptomatic gCJD cases associated with the E200K and V210I mutations.

In contrast with longitudinal data from neuroaxonal/synaptic damage markers such as t-tau, 14-3-3, Nfl, and $\alpha$-syn, which present marginal or absent alterations of their CSF 
concentrations along disease progression $[33,59,77]$, the progressive neuroinflammatory profile in SCJD brain may explain increased CSF sTREM2 concentrations in follow-up LPs, suggesting a potential role for STREM2 as a candidate marker of microglial activation. The observation that CSF and plasma STREM2 are not associated with disease duration in SCJD precludes a prognostic value for STREM2.

In conclusion, we have found expression of TREM 2 in a subpopulation of microglial cells in human SCJD brains, with increased mRNA and protein levels in brain compared to controls. Further, we have shown that CSF and plasma sTREM2 have diagnostic accuracy in the discrimination of SCJD. We may speculate that sTREM2 quantification in CSF may be useful for complementary diagnostic purposes. However, the assessment of TREM2 levels in the CSF in other neurological diseases, that may represent clinical diagnosis difficulties with $\mathrm{CJD}$, is a pending condition. Unfortunately, adequate numbers of samples from cases with paraneoplastic encephalitis, other rare encephalitis, and rapid forms of non-prionic dementia were not available in the present study. Finally, our data highlight the importance of molecular neuropathology assessment in the context of biomarker research toward understanding of the aetiology of the alterations detected in biological fluids, which in turn may be relevant in the interpretation of the translational value of biomarker profiles.

Supplementary Information The online version contains supplementary material available at https://doi.org/10.1007/s00401-021-02296-1.

Acknowledgements We thank the HUB-ICO-IDIBELL-Biobank and the CERCA Programme of the Generalitat de Catalunya for institutional support. This study was funded by the Instituto Carlos III (Grants Number CP16/00041 and PI19/00144) to FL. This project was also funded by la Fundació La Marató de TV3 (Grants No. 201821-30, 201821-31 and 201821-32 to FL, JCE and EV, respectively) and cofinanced by ERDF under the programme Interreg Poctefa: RedPrion $148 / 16$ to IF. AVP is supported by the Beatriu de Pinós programme (2018-BP-00129) from the Ministry of Business and Knowledge of the Government of Catalonia, and cofunded by the EU Horizon 2020 programme under an MSCA grant agreement (801370). HZ is a Wallenberg Scholar supported by grants from the Swedish Research Council (\#2018-02532), the European Research Council (\#681712), Swedish State Support for Clinical Research (\#ALFGBG-720931), the Alzheimer Drug Discovery Foundation (ADDF), USA (\#201809-2016862), and the UK Dementia Research Institute at UCL. KB is supported by the Swedish Research Council (\#2017-00915), the Alzheimer Drug Discovery Foundation (ADDF), USA (\#RDAPB-201809-2016615), the Swedish Alzheimer Foundation (\#AF-742881), Hjärnfonden, Sweden (\#FO2017-0243), the Swedish state under the agreement between the Swedish government and the County Councils, the ALF-agreement (\#ALFGBG-715986), and the European Union Joint Programme for Neurodegenerative Disorders (JPND2019-466-236). AL is supported by the Ministero della Salute, Italy, for the national surveillance of Creutzfeldt-Jakob disease. This research was also supported in part by the Alberta Synergies in Alzheimer's and Related Disorders (SynAD) programme which is funded by the Alzheimer Society of Alberta and Northwest Territories through their Hope for Tomorrow programme and the University Hospital Foundation. SynAD operates in partnership with the Neuroscience and Mental Health Institute at the University of
Alberta. JADR was supported by grants from the Spanish Ministry of Science, Innovation and Universities (MICINN/FEDER) (RTI2018099773-B-I00), the CERCA Programme, and by the Commission for Universities and Research of the Department of Innovation, Universities, and Enterprise of the Generalitat de Catalunya (SGR2017-648) and CIBERNED (CMED2018-2).

Author contributions FL designed the study. DD-L, NK, AV-P, OL-P, KT, MS, JEC, PA-B, HP, and VS performed the experiments and analysed the data. PH, AL, MC, JR, HZ, KB, JAR, RS-V, BM, IF, IZ, and FL contributed to clinical and biochemical data acquisition, interpretation, and sampling. AM-M, JCE, and JMT performed animal experimentation. EV contributed to technical and analytical expertise. FL, IF and IZ drafted the manuscript; all authors critically revised the manuscript and approved its contents before submission.

Funding We thank the HUB-ICO-IDIBELL-Biobank and the CERCA Programme of the Generalitat de Catalunya for institutional support. This study was funded by the Instituto Carlos III (Grants Number CP16/00041 and PI19/00144) to FL. This project was also funded by la Fundació La Marató de TV3 (Grants No. 201821-30, 201821-31 and 201821-32 to FL, JCE and EV, respectively) and by the Fondo Europeo de Desarrollo Regional (FEDER) through the Interreg V-A España-Francia-Andorra (POCTEFA 2014-2020) programme (at $65 \%)$ to IF. AVP is supported by the Beatriu de Pinós programme (2018-BP-00129) from the Ministry of Business and Knowledge of the Government of Catalonia, and cofunded by the EU Horizon 2020 programme under an MSCA grant agreement (801370). HZ is a Wallenberg Scholar supported by grants from the Swedish Research Council (\#2018-02532), the European Research Council (\#681712), Swedish State Support for Clinical Research (\#ALFGBG-720931), the Alzheimer Drug Discovery Foundation (ADDF), USA (\#201809-2016862), and the UK Dementia Research Institute at UCL. KB is supported by the Swedish Research Council (\#2017-00915), the Alzheimer Drug Discovery Foundation (ADDF), USA (\#RDAPB-201809-2016615), the Swedish Alzheimer Foundation (\#AF-742881), Hjärnfonden, Sweden (\#FO2017-0243), the Swedish state under the agreement between the Swedish government and the County Councils, the ALF-agreement (\#ALFGBG-715986), and the European Union Joint Programme for Neurodegenerative Disorders (JPND2019-466-236). AL is supported by the Ministero della Salute, Italy, for the national surveillance of Creutzfeldt-Jakob disease. This research was also supported in part by the Alberta Synergies in Alzheimer's and Related Disorders (SynAD) programme which is funded by the Alzheimer Society of Alberta and Northwest Territories through their Hope for Tomorrow programme and the University Hospital Foundation. SynAD operates in partnership with the Neuroscience and Mental Health Institute at the University of Alberta. JADR was supported by grants from the Spanish Ministry of Science, Innovation and Universities (MICINN/FEDER) (RTI2018099773-B-I00), the CERCA Programme, and by the Commission for Universities and Research of the Department of Innovation, Universities, and Enterprise of the Generalitat de Catalunya (SGR2017-648) and CIBERNED (CMED2018-2).

\section{Declarations}

Conflict of interest HZ has served on scientific advisory boards for Denali, Roche Diagnostics, Wave, Samumed, Siemens Healthineers, Pinteon Therapeutics, and $\operatorname{CogRx}$, has given lectures in symposia sponsored by Fujirebio, Alzecure, and Biogen, and is a co-founder of Brain Biomarker Solutions in Gothenburg AB (BBS), which is a part of the GU Ventures Incubator Programme. KB has served as a consultant, on advisory boards, and on data monitoring committees for Abcam, Axon, Biogen, Julius Clinical, Lilly, MagQu, Novartis, Roche 
Diagnostics, and Siemens Healthineers, and is a co-founder of Brain Biomarker Solutions in Gothenburg AB (BBS), which is a part of the GU Ventures Incubator Programme.

Human sample acquisition Brain tissue was obtained from the Institute of Neuropathology HUB-ICO-IDIBELL Biobank and the Hospital Clinic de Barcelona-IDIBAPS Neurological Tissue Bank following the Spanish legislation guidelines on this matter (Real Decreto de Bioban$\cos 1716 / 2011$ ) and approval of the local ethics committees. CSF samples were collected at the following centres: (1) Clinical Dementia Centre and the National Reference Centre for CJD Surveillance at the University Medical Centre of Göttingen (Germany); (2) Alzheimer's Disease and Other Cognitive Disorders Unit, Hospital Clínic de Barcelona, Barcelona (Spain); (3) Multiple Sclerosis Unit Neurology Service, Bellvitge University Hospital, Hospitalet de Llobregat, Spain; (4) National Centre of Microbiology-Instituto de Salud Carlos III, Madrid, Spain; and (5) Istituto Superiore di Sanità, Rome, Italy. Blood was collected at the Department of Transfusion Medicine (healthy controls) and at the Department of Neurology (sCJD cases) of the University Medical Centre Göttingen (Germany) under standardized pre-analytical conditions.

Animal experimentation We conducted animal experiments in accordance with the Code for Methods and Welfare Considerations in Behavioural Research with Animals (Directive 2010/63/EU) and made every effort to minimize suffering. Experiments developed in the Centro de Investigación en Sanidad Animal-Instituto Nacional de Investigación y Tecnología Agraria y Alimentaria (Madrid, Spain) were evaluated by the Committee on the Ethics of Animal Experiments of the Instituto Nacional de Investigación y Tecnología Agraria y Alimentaria and approved by the General Directorate of the Madrid Community Government (permit no. PROEX 094/18).

Open Access This article is licensed under a Creative Commons Attribution 4.0 International License, which permits use, sharing, adaptation, distribution and reproduction in any medium or format, as long as you give appropriate credit to the original author(s) and the source, provide a link to the Creative Commons licence, and indicate if changes were made. The images or other third party material in this article are included in the article's Creative Commons licence, unless indicated otherwise in a credit line to the material. If material is not included in the article's Creative Commons licence and your intended use is not permitted by statutory regulation or exceeds the permitted use, you will need to obtain permission directly from the copyright holder. To view a copy of this licence, visit http://creativecommons.org/licenses/by/4.0/.

\section{References}

1. Abdelhak A, Hottenrott T, Morenas-Rodríguez E, Suárez-Calvet M, Zettl UK, Haass C et al (2019) Glial activation markers in CSF and serum from patients with primary progressive multiple sclerosis: potential of serum GFAP as disease severity marker? Front Neurol 10:280. https://doi.org/10.3389/fneur.2019.00280. eCollection

2. Abu-Rumeileh S, Steinacker P, Polischi B, Mammana A, Bartoletti-Stella A, Oeckl P et al (2019) CSF biomarkers of neuroinflammation in distinct forms and subtypes of neurodegenerative dementia. Alzheimer's Res Ther 12:2. https://doi.org/10.1186/ s13195-019-0562-4

3. Aguzzi A, Sigurdson C, Heikenwalder M (2007) Molecular mechanisms of prion pathogenesis. Annu Rev Pathol Mech Dis 3:11-40. https://doi.org/10.1146/annurev.pathol.3.121806.154326
4. Bekris LM, Khrestian M, Dyne E, Shao Y, Pillai J, Rao S et al (2018) Soluble TREM2 and biomarkers of central and peripheral inflammation in neurodegenerative disease. J Neuroimmunol 319:19-27. https://doi.org/10.1016/j.jneuroim.2018.03.003

5. Braak H, Braak E (1991) Neuropathological stageing of Alzheimer-related changes. Acta Neuropathol 82:239-259. https://doi. org/10.1007/BF00308809

6. Braak H, Alafuzoff I, Arzberger T, Kretzschmar H, Del Tredici K (2006) Staging of Alzheimer disease-associated neurofibrillary pathology using paraffin sections and immunocytochemistry. Acta Neuropathol 112(4):389-404. https://doi.org/10.1007/ s00401-006-0127-z

7. Cady J, Koval ED, Benitez BA, Zaidman C, Jockel-Balsarotti J, Allred $\mathrm{P}$ et al (2014) TREM2 variant p. R47H as a risk factor for sporadic amyotrophic lateral sclerosis. JAMA Neurol 71:449-453. https://doi.org/10.1001/jamaneurol.2013.6237

8. Capellari S, Strammiello R, Saverioni D, Kretzschmar H, Parchi P (2011) Genetic Creutzfeldt-Jakob disease and fatal familial insomnia: Insights into phenotypic variability and disease pathogenesis. Acta Neuropathol 121:21-37. https://doi.org/10.1007/ s00401-010-0760-4

9. Cassard H, Huor A, Espinosa JC, Douet JY, Lugan S, Aron N et al (2020) Prions from sporadic Creutzfeldt-Jakob disease patients propagate as strain mixtures. MBio 11:1-19. https://doi.org/10. 1128/mBio.00393-20

10. Cassard H, Torres JM, Lacroux C, Douet JY, Benestad SL, Lantier F et al (2014) Evidence for zoonotic potential of ovine scrapie prions. Nat Commun 5(5):5821. https://doi.org/10.1038/ncomm s6821

11. Chen S, Peng J, Sherchan P, Ma Y, Xiang S, Yan F et al (2020) TREM2 activation attenuates neuroinflammation and neuronal apoptosis via PI3K/Akt pathway after intracerebral hemorrhage in mice. J Neuroinflammation 17(1):168. https://doi.org/10.1186/ s12974-020-01853-x

12. Cheng-Hathaway PJ, Reed-Geaghan EG, Jay TR, Casali BT, Bemiller SM, Puntambekar SS et al (2018) The Trem2 R47H variant confers loss-of-function-like phenotypes in Alzheimer's disease. Mol Neurodegener 13:29. https://doi.org/10.1186/ s13024-018-0262-8

13. Cramm M, Schmitz M, Karch A, Mitrova E, Kuhn F, Schroeder B et al (2016) Stability and reproducibility underscore utility of RT-QuIC for diagnosis of Creutzfeldt-Jakob disease. Mol Neurobiol 53:1896-1904. https://doi.org/10.1007/ s12035-015-9133-2

14. Deczkowska A, Weiner A, Amit I (2020) The physiology, pathology, and potential therapeutic applications of the TREM2 signaling pathway. Cell 181:1207-1217. https://doi.org/10.1016/j.cell. 2020.05.003

15. Fahrenhold M, Rakic S, Classey J, Brayne C, Ince PG, Nicoll JAR et al (2018) TREM2 expression in the human brain: a marker of monocyte recruitment? Brain Pathol 28(5):595-602. https://doi. org/10.1111/bpa.12564

16. Ferrer I (2014) Brain banking. Encyclopedia of the Neurological Sciences. Academic Press, pp 467-473

17. Gambetti P, Kong Q, Zou W, Parchi P, Chen SG (2003) Sporadic and familial CJD: classification and characterisation. Br Med Bull 66:213-239. https://doi.org/10.1093/bmb/66.1.213

18. Geschwind MD (2015) Prion diseases HHS public access. Contin Neuroinfec Dis 6:1612-1638. https://doi.org/10.1212/CON.00000 00000000251

19. Gmitterová K, Heinemann U, Bodemer M, Krasnianski A, Meissner B, Kretzschmar HA et al (2009) 14-3-3 CSF levels in sporadic Creutzfeldt-Jakob disease differ across molecular subtypes. Neurobiol Aging 30:1842-1850. https://doi.org/10.1016/j.neurobiola ging.2008.01.007 
20. Guerreiro R, Bilgic B, Guven G, Brás J, Rohrer J, Lohmann E et al (2013) A novel compound heterozygous mutation in TREM2 found in a Turkish frontotemporal dementia-like family. Neurobiol Aging 34:2890.e1-5. https://doi.org/10.1016/j.neurobiolaging. 2013.06.005

21. Guerreiro R, Wojtas A, Bras J, Carrasquillo M, Rogaeva E, Majounie E et al (2013) TREM2 variants in Alzheimer's disease. N Engl J Med 368(2):117-127. https://doi.org/10.1056/NEJMo a1211851

22. Henjum K, Almdahl IS, Årskog V, Minthon L, Hansson O, Fladby $\mathrm{T}$ et al (2016) Cerebrospinal fluid soluble TREM2 in aging and Alzheimer's disease. Alzheimer's Res Ther 8(1):17. https://doi. org/10.1186/s13195-016-0182-1

23. Hermann P, Laux M, Glatzel M, Matschke J, Knipper T, Goebel $S$ et al (2018) Validation and utilization of amended diagnostic criteria in Creutzfeldt-Jakob disease surveillance. Neurology 91(4):e331-e338. https://doi.org/10.1212/WNL.0000000000 005860

24. Heslegrave A, Heywood W, Paterson R, Magdalinou N, Svensson J, Johansson P et al (2016) Increased cerebrospinal fluid soluble TREM2 concentration in Alzheimer's disease. Mol Neurodegener 11:3. https://doi.org/10.1186/s13024-016-0071-x

25. Jesse S, Steinacker P, Cepek L, Arnim CV, Tumani H, Lehnert $S$ et al (2009) Glial fibrillary acidic protein and protein S-100B: different concentration pattern of glial proteins in cerebrospinal fluid of patients with Alzheimer's disease and Creutzfeldt-Jakob disease. J Alzheimer's Dis 17(3):541-551. https://doi.org/10. 3233/JAD-2009-1075

26. Jonsson T, Stefansson H, Steinberg S, Jonsdottir I, Jonsson PV, Snaedal J et al (2013) Variant of TREM2 associated with the risk of Alzheimer's disease. N Engl J Med 368(2):107-116. https:// doi.org/10.1056/NEJMoa1211103

27. Karch A, Hermann P, Ponto C, Schmitz M, Arora A, Zafar S et al (2015) Cerebrospinal fluid tau levels are a marker for molecular subtype in sporadic Creutzfeldt-Jakob disease. Neurobiol Aging 36:1964-1968. https://doi.org/10.1016/j.neurobiolaging.2015.01. 021

28. Kiialainen A, Hovanes K, Paloneva J, Kopra O, Peltonen L (2005) Dap12 and Trem2, molecules involved in innate immunity and neurodegeneration, are co-expressed in the CNS. Neurobiol Dis 18(2):314-322. https://doi.org/10.1016/j.nbd.2004.09.007

29. Kleinberger G, Yamanishi Y, Suárez-Calvet M, Czirr E, Lohmann E, Cuyvers E et al (2014) TREM2 mutations implicated in neurodegeneration impair cell surface transport and phagocytosis. Sci Transl Med 6(243):243. https://doi.org/10.1126/scitranslmed. 3009093

30. Kovacs GG, Andreasson U, Liman V, Regelsberger G, Lutz MI, Danics K et al (2017) Plasma and cerebrospinal fluid tau and neurofilament concentrations in rapidly progressive neurological syndromes: a neuropathology-based cohort. Eur J Neurol 24(11):1326-e77. https://doi.org/10.1111/ene.13389

31. Kovács GG, Puopolo M, Ladogana A, Pocchiari M, Budka H, van Duijn C et al (2005) Genetic prion disease: the EUROCJD experience. Hum Genet 118:166-174. https://doi.org/10.1007/ s00439-005-0020-1

32. Ladogana A, Sanchez-Juan P, Mitrova E, Green A, CuadradoCorrales N, Sanchez-Valle R et al (2009) Cerebrospinal fluid biomarkers in human genetic transmissible spongiform encephalopathies. J Neurol 256:1620-1628. https://doi.org/10.1007/ s00415-009-5163-x

33. Llorens F, Kruse N, Karch A, Schmitz M, Zafar S, Gotzmann N et al (2017) Validation of $\alpha$-synuclein as a CSF biomarker for sporadic Creutzfeldt-Jakob disease. Mol Neurobiol 55(3):2249-2257. https://doi.org/10.1007/s12035-017-0479-5

34. Llorens F, Lopez-Gonzalez I, Thune K, Carmona M, Zafar S, Andeoletti O et al (2014) Subtype and regional-specific neuroinflammation in sporadic Creutzfeldt-Jakob disease. Front Aging Neurosci 6:198. https://doi.org/10.3389/fnagi.2014.00198

35. Llorens F, Rübsamen N, Hermann P, Schmitz M, Villar-Piqué A, Goebel S et al (2020) A prognostic model for overall survival in sporadic Creutzfeldt-Jakob disease. Alzheimer's Dement 16(10):1438-1447. https://doi.org/10.1002/alz.12133

36. Llorens F, Schmitz M, Karch A, Cramm M, Lange P, Gherib K et al (2015) Comparative analysis of cerebrospinal fluid biomarkers in the differential diagnosis of neurodegenerative dementia. Alzheimers Dement 2(5):577-89. https://doi.org/10.1016/j.jalz. 2015.10.009

37. Llorens F, Thüne K, Tahir W, Kanata E, Diaz-Lucena D, Xanthopoulos K et al (2017) YKL-40 in the brain and cerebrospinal fluid of neurodegenerative dementias. Mol Neurodegener 12(1):83. https://doi.org/10.1186/s13024-017-0226-4

38. Llorens F, Villar-piqué A, Hermann P, Schmitz M, Calero O, Stehmann C et al (2020) Diagnostic accuracy of prion disease biomarkers in iatrogenic Creutzfeldt-Jakob disease. Biomolecules 10(2):290. https://doi.org/10.3390/biom10020290

39. Llorens F, Zafar S, Ansoleaga B, Shafiq M, Blanco R, Carmona M et al (2015) Subtype and regional regulation of prion biomarkers in sporadic Creutzfeldt-Jakob disease. Neuropathol Appl Neurobiol 41:631-645. https://doi.org/10.1111/nan.12175

40. Llorens F, Zarranz JJ, Fischer A, Zerr I, Ferrer I (2017) Fatal familial insomnia: clinical aspects and molecular alterations. Curr Neurol Neurosci Rep 17(4):30. https://doi.org/10.1007/ s11910-017-0743-0

41. Lue LF, Schmitz CT, Serrano G, Sue LI, Beach TG, Walker DG (2015) TREM2 Protein expression changes correlate with Alzheimer's disease neurodegenerative pathologies in post-mortem temporal cortices. Brain Pathol 25(4):469-80. https://doi.org/10. 1111/bpa.12190

42. Ma L, Allen M, Sakae N, Ertekin-Taner N, Graff-Radford NR, Dickson DW et al (2016) Expression and processing analyses of wild type and pR47H TREM2 variant in Alzheimer's disease brains. Mol Neurodegener 11(1):72. https://doi.org/10.1186/ s13024-016-0137-9

43. Mackenzie IRA, Neumann M, Baborie A, Sampathu DM, Du Plessis D, Jaros E et al (2011) A harmonized classification system for FTLD-TDP pathology. Acta Neuropathol 122(1):111-3. https://doi.org/10.1007/s00401-011-0845-8

44. McGuire LI, Peden AH, Orrú CD, Wilham JM, Appleford NE, Mallinson $G$ et al (2012) Real time quaking-induced conversion analysis of cerebrospinal fluid in sporadic Creutzfeldt-Jakob disease. Ann Neurol 72:278-285. https://doi.org/10.1002/ana. 23589

45. McKhann GM, Knopman DS, Chertkow H, Hyman BT, Jack CR, Kawas CH et al (2011) The diagnosis of dementia due to Alzheimer's disease: recommendations from the National Institute on Aging-Alzheimer's Association workgroups on diagnostic guidelines for Alzheimer's disease. Alzheimers Dement 7:263-269. https://doi.org/10.1016/j.jalz.2011.03.005

46. Minikel EV, Zhao HT, Le J, O'Moore J, Pitstick R, Graffam S et al (2020) Prion protein lowering is a disease-modifying therapy across prion strains, disease stages, and endpoints. Nucleic Acids Res 48(19):10615-10631. https://doi.org/10.1093/nar/gkaa616

47. Montagna P, Cortelli P, Avoni P, Tinuper P, Plazzi G, Gallassi R et al (1998) Clinical features of fatal familial insomnia: phenotypic variability in relation to a polymorphism at codon 129 of the prion protein gene. Brain Pathol 8:515-520. https://doi.org/ 10.1111/j.1750-3639.1998.tb00172.x

48. Öhrfelt A, Axelsson M, Malmeström C, Novakova L, Heslegrave A, Blennow K et al (2016) Soluble TREM-2 in cerebrospinal fluid from patients with multiple sclerosis treated with natalizumab or mitoxantrone. Mult Scler 22:1587-1595. https://doi.org/10.1177/ 1352458515624558 
49. Parchi P, De Boni L, Saverioni D, Cohen ML, Ferrer I, Gambetti $P$ et al (2012) Consensus classification of human prion disease histotypes allows reliable identification of molecular subtypes: An inter-rater study among surveillance centres in Europe and USA. Acta Neuropathol 124:517-529. https://doi.org/10.1007/ s00401-012-1002-8

50. Parchi P, Giese A, Capellari S, Brown P, Schulz-Schaeffer W, Windl O et al (1999) Classification of sporadic Creutzfeldt-Jakob disease based on molecular and phenotypic analysis of 300 subjects. Ann Neurol 46:224-233

51. Piccio L, Buonsanti C, Cella M, Tassi I, Schmidt RE, Fenoglio C et al (2008) Identification of soluble TREM-2 in the cerebrospinal fluid and its association with multiple sclerosis and CNS inflammation. Brain 131:3081-3091. https://doi.org/10.1093/brain/ awn217

52. Piccio L, Deming Y, Del-Aguila JL, Ghezzi L, Holtzman DM, Fagan AM et al (2016) Cerebrospinal fluid soluble TREM2 is higher in Alzheimer disease and associated with mutation status. Acta Neuropathol 131:925-933. https://doi.org/10.1007/ s00401-016-1533-5

53. Pinheiro J, Bates D, DebRoy S, Sarkar D, R Core Team (2020) nlme: Linear and nonlinear mixed effects models. R package version 3.1-152, https://CRAN.R-project.org/package=nlme

54. Puoti G, Bizzi A, Forloni G, Safar JG, Tagliavini F, Gambetti P (2012) Sporadic human prion diseases: molecular insights and diagnosis. Lancet Neurol 11:618-628. https://doi.org/10.1016/ S1474-4422(12)70063-7

55. Rauchmann BS, Schneider-Axmann T, Alexopoulos P, Perneczky R, Alzheimer's Disease Neuroimaging Initiative (2019) CSF soluble TREM2 as a measure of immune response along the Alzheimer's disease continuum. Neurobiol Aging 74:182-190. https:// doi.org/10.1016/j.neurobiolaging.2018.10.022

56. Rayaprolu S, Mullen B, Baker M, Lynch T, Finger E, Seeley WW et al (2013) TREM2 in neurodegeneration: evidence for association of the $\mathrm{pR} 47 \mathrm{H}$ variant with frontotemporal dementia and Parkinson's disease. Mol Neurodegener. https://doi.org/10.1186/ 1750-1326-8-19

57. Robin X, Turck N, Hainard A, Tiberti N, Lisacek F, Sanchez JC et al (2011) pROC: an open-source package for R and S+ to analyze and compare ROC curves. BMC Bioinformatics 12:77. https://doi.org/10.1186/1471-2105-12-77

58. Sanchez-Juan P, Green A, Ladogana A, Cuadrado-Corrales N, Sáanchez-Valle R, Mitrováa E et al (2006) CSF tests in the differential diagnosis of Creutzfeldt-Jakob disease. Neurology 67:637643. https://doi.org/10.1212/01.wnl.0000230159.67128.00

59. Sanchez-Juan P, Sánchez-Valle R, Green A, Ladogana A, Cuadrado-Corrales N, Mitrová E et al (2007) Influence of timing on CSF tests value for Creutzfeldt-Jakob disease diagnosis. J Neurol 254:901-906. https://doi.org/10.1007/s00415-006-0472-9

60. Schlepckow K, Kleinberger G, Fukumori A, Feederle R, Lichtenthaler SF, Steiner H et al (2017) An Alzheimer-associated TREM2 variant occurs at the ADAM cleavage site and affects shedding and phagocytic function. EMBO Mol Med 9:1356-1365. https://doi.org/10.15252/emmm.201707672

61. Schmid CD, Sautkulis LN, Danielson PE, Cooper J, Hasel KW, Hilbush BS et al (2002) Heterogeneous expression of the triggering receptor expressed on myeloid cells- 2 on adult murine microglia. J Neurochem 83(6):1309-1320. https://doi.org/10.1046/j. 1471-4159.2002.01243.x

62. Schmitz M, Ebert E, Stoeck K, Karch A, Collins S, Calero M et al (2016) Validation of 14-3-3 Protein as a marker in sporadic Creutzfeldt-Jakob disease diagnostic. Mol Neurobiol 53:2189_ 2199. https://doi.org/10.1007/s12035-015-9167-5

63. Slattery CF, Beck JA, Harper L, Adamson G, Abdi Z, Uphill J et al (2014) R47H TREM2 variant increases risk of typical early-onset Alzheimer's disease but not of prion or frontotemporal dementia.
Alzheimers Dement 10:602-608.e4. https://doi.org/10.1016/j.jalz. 2014.05.1751

64. Song WM, Joshita S, Zhou Y, Ulland TK, Gilfillan S, Colonna M (2018) Humanized TREM2 mice reveal microglia-intrinsic and -extrinsic effects of R47H polymorphism. J Exp Med 215(3):745760. https://doi.org/10.1084/jem.20171529

65. Staffaroni AM, Kramer AO, Casey M, Kang H, Rojas JC, Orrú CD et al (2019) Association of blood and cerebrospinal fluid tau level and other biomarkers with survival time in sporadic CreutzfeldtJakob disease. JAMA Neurol 76(8):969-977. https://doi.org/10. 1001/jamaneurol.2019.1071

66. Steinacker P, Blennow K, Halbgebauer S, Shi S, Ruf V, Oeckl P et al (2016) Neurofilaments in blood and CSF for diagnosis and prediction of onset in Creutzfeldt-Jakob disease. Sci Rep 6:38737. https://doi.org/10.1038/srep38737

67. Suárez-Calvet M, Kleinberger G, Araque Caballero MÁ, Brendel M, Rominger A, Alcolea D et al (2016) sTREM 2 cerebrospinal fluid levels are a potential biomarker for microglia activity in early-stage Alzheimer's disease and associate with neuronal injury markers. EMBO Mol Med 8(5):466-476. https://doi.org/ 10.15252/emmm.201506123

68. Suárez-Calvet M, Morenas-Rodríguez E, Kleinberger G, Schlepckow K, Araque Caballero MÁ, Franzmeier N et al (2019) Early increase of CSF sTREM2 in Alzheimer's disease is associated with tau related-neurodegeneration but not with amyloid- $\beta$ pathology. Mol Neurodegener 14(1):1. https://doi.org/10.1186/s13024018-0301-5 (PMID: 30630532)

69. Thompson AJ, Baranzini SE, Geurts J, Hemmer B, Ciccarelli O (2018) Multiple sclerosis. Lancet 391(10130):1622-1636. https:// doi.org/10.1016/S0140-6736(18)30481-1

70. Thompson AGB, Mead SH (2019) Review: fluid biomarkers in the human prion diseases. Mol Cell Neurosci 97:81-92. https:// doi.org/10.1016/j.mcn.2018.12.003

71. Vallabh SM, Nobuhara CK, Llorens F, Zerr I, Parchi P, Capellari $S$ et al (2019) Prion protein quantification in human cerebrospinal fluid as a tool for prion disease drug development. Proc Natl Acad Sci USA 116:7793-7798. https://doi.org/10.1073/pnas.19019 47116

72. Villar-Piqué A, Schmitz M, Hermann P, Goebel S, Bunck T, Varges D et al (2019) Plasma YKL-40 in the spectrum of neurodegenerative dementia. J Neuroinflammation 16(1):145. https://doi. org/10.1186/s12974-019-1531-3

73. World Health Organisation (2003) WHO manual for surveillance of human transmissible spongiform encephalopathies including variant Creutzfeldt-Jakob disease. WHO Man Surveill Hum Transm spongiform Enceph, Geneva

74. Wu K, Byers DE, Jin X, Agapov E, Alexander-Brett J, Patel AC et al (2015) TREM-2 promotes macrophage survival and lung disease after respiratory viral infection. J Exp Med 212(5):681-97. https://doi.org/10.1084/jem.20141732

75. Zerr I, Bodemer M, Gefeller O, Otto M, Poser S, Wiltfang J et al (1998) Detection of 14-3-3 protein in the cerebrospinal fluid supports the diagnosis of Creutzfeldt-Jakob disease. Ann Neurol 43:32-40. https://doi.org/10.1002/ana.410430109

76. Zerr I, Kallenberg K, Summers DM, Romero C, Taratuto A, Heinemann U et al (2009) Updated clinical diagnostic criteria for sporadic Creutzfeldt-Jakob disease. Brain 132:2659-2668. https:// doi.org/10.1093/brain/awp191

77. Zerr I, Schmitz M, Karch A, Villar-Piqué A, Kanata E, Golanska E et al (2018) Cerebrospinal fluid neurofilament light levels in neurodegenerative dementia: Evaluation of diagnostic accuracy in the differential diagnosis of prion diseases. Alzheimer's Dement 14(6):751-763. https://doi.org/10.1016/j.jalz.2017.12.008

78. Zetterberg $\mathrm{H}$ (2017) Fluid biomarkers for microglial activation and axonal injury in multiple sclerosis. Acta Neurol Scand 201:15-17. https://doi.org/10.1111/ane.12845 
79. Zhong L, Xu Y, Zhuo R, Wang T, Wang K, Huang R et al (2019) Soluble TREM2 ameliorates pathological phenotypes by modulating microglial functions in an Alzheimer's disease model. Nat Commun 10(1):1365. https://doi.org/10.1038/ s41467-019-09118-9

80. Zhu C, Herrmann US, Li B, Abakumova I, Moos R, Schwarz $P$ et al (2015) Triggering receptor expressed on myeloid cells-2 is involved in prion-induced microglial activation but does not contribute to prion pathogenesis in mouse brains. Neurobiol Aging 36(5):1994-2003. https://doi.org/10.1016/j.neurobiolaging. 2015.02.019

Publisher's Note Springer Nature remains neutral with regard to jurisdictional claims in published maps and institutional affiliations.

\section{Authors and Affiliations}

\section{Daniela Diaz-Lucena ${ }^{1,2} \cdot$ Niels Kruse $^{3}$ - Katrin Thüne ${ }^{4}$ - Matthias Schmitz ${ }^{4,5}$ - Anna Villar-Piqué ${ }^{1,2}$. Jose Eriton Gomes da Cunha ${ }^{6}$. Peter Hermann ${ }^{4}$. Óscar López-Pérez ${ }^{1,2}$ • Pol Andrés-Benito ${ }^{1,2} \cdot$ Anna Ladogana $^{7}$. Miguel Calero ${ }^{8,9} \cdot$ Enric Vidal $^{10}$. Joachim Riggert ${ }^{11} \cdot$ Hailey Pineau ${ }^{12}$. Valerie Sim ${ }^{12} \cdot$ Henrik Zetterberg $^{13,14,15,16}$. Kaj Blennow ${ }^{13,14}$ • Jose Antonio del Río ${ }^{17,18,19,20}$ • Alba Marín-Moreno ${ }^{21}$ • Juan Carlos Espinosa ${ }^{21}$. Juan María Torres ${ }^{21} \cdot$ Raquel Sánchez-Valle $^{22} \cdot$ Brit Mollenhauer $^{23,24} \cdot$ Isidre Ferrer $^{1,2,25}$ (D) Inga Zerr ${ }^{4,5}$. Franc Llorens ${ }^{1,2,4}$}

1 Network Center for Biomedical Research in Neurodegenerative Diseases (CIBERNED), L'Hospitalet de Llobregat, Spain

2 Bellvitge Biomedical Research Institute (IDIBELL), L'Hospitalet de Llobregat, Spain

3 University Medical Center Göttingen, Institute of Neuropathology, Göttingen, Germany

4 Department of Neurology, University Medical Center Göttingen, Gern August University, Robert Koch Strasse 40, 37075 Göttingen, Germany

5 German Center for Neurodegenerative Diseases (DZNE), Göttingen, Germany

6 Keizo Asami Laboratory (LIKA), Universidade Federal de Pernambuco (UFPE), Recife, Brazil

7 Department of Neurosciences, Istituto Superiore Di Sanità, Rome, Italy

8 Alzheimer Disease Research Unit, CIEN Foundation, Chronic Disease Programme, Queen Sofia Foundation Alzheimer Center, Instituto de Salud Carlos III, Madrid, Spain

9 Network Center for Biomedical Research in Neurodegenerative Diseases (CIBERNED), Madrid, Spain

10 Centre de Recerca en Sanitat Animal, Campus Universitat Autònoma de Barcelona, Institut de Recerca I Tecnologia Agroalimentàries, Bellaterra, Spain

11 Department of Transfusion Medicine, University Medical School, Göttingen, Germany

12 Department of Medicine-Division of Neurology, Centre for Prions and Protein Folding Diseases, University of Alberta, Edmonton, Canada

13 Department of Psychiatry and Neurochemistry, The Sahlgrenska Academy at the University of Gothenburg, Institute of Neuroscience and Physiology, Mölndal, Sweden
14 Clinical Neurochemistry Laboratory, Sahlgrenska University Hospital, Mölndal, Sweden

15 Department of Neurodegenerative Disease, UCL Institute of Neurology, London, UK

16 UK Dementia Research Institute, London, UK

17 Molecular and Cellular Neurobiotechnology, Scientific Park of Barcelona, Institute for Bioengineering of Catalonia (IBEC), The Barcelona Institute for Science and Technology (BIST), Barcelona, Spain

18 Department of Cell Biology, Physiology and Immunology, Faculty of Biology, University of Barcelona, Barcelona, Spain

19 Network Center for Biomedical Research in Neurodegenerative Diseases (CIBERNED), Barcelona, Spain

20 University of Barcelona, Institute of Neuroscience, Barcelona, Spain

21 Centro de Investigación en Sanidad Animal, CISA-INIA, Madrid, Spain

22 Alzheimer's Disease and Other Cognitive Disorders Unit, Neurology Department, Hospital Clinic de Barcelona, Institut D'Investigacions Biomediques August Pi I Sunyer (IDIBAPS), Barcelona, Spain

23 Paracelsus-Elena Klinik, Kassel, Germany

24 Department of Neurology, University Medical Centre Göttingen, Göttingen, Germany

25 Department of Pathology and Experimental Therapeutics, Hospitalet de Llobregat, University of Barcelona, Feixa Llarga S/N, 08907 Barcelona, Spain 